\title{
Integer and half-integer channel spins for elastic scattering cross sections
}

\author{
A. S. Tkachenko, ${ }^{1,2}$ R. Ya. Kezerashvili, ${ }^{3,4}$ N. A. Burkova, ${ }^{2}$ S. B. Dubovichenko ${ }^{1,2}$ \\ ${ }^{1}$ Fesenkov Astrophysical Institute "NCSRT" ASA MDASI RK, 050020, Almaty, Kazakhstan \\ ${ }^{2}$ al-Farabi Kazakh National University, 050040, Almaty, Kazakhstan \\ ${ }^{3}$ New York City College of Technology, City University of New York, Brooklyn, NY 11201, USA \\ ${ }^{4}$ Graduate School and University Center, City University of New York, New York 10016, USA
}

(Dated: May 7, 2019)

\begin{abstract}
We present the analytical expressions for the differential cross sections and independent partial amplitudes for elastic scattering of nuclear particles for channels with a spin value of $1 / 2,1,3 / 2,2$ and $5 / 2$. The independent partial amplitudes are presented for arbitrary orbital angular momentum $l$ and taking into account spin-orbit splitting. The analytical expressions allow one to carry out full phase shift analyses using experimental data for differential cross sections for processes with channel spins $1 / 2,1,3 / 2,2$ and $5 / 2$.
\end{abstract}

\section{INTRODUCTION}

One of the most important problems in the theory of nuclear reactions and nuclear scattering is a construction of potentials for a nucleon-nucleon, nucleon-nuclear and nuclear-nuclear interactions. These potentials are used for calculation of any nuclear characteristics, for example, the binding energies of the nuclei, the properties of their bound states, cross sections, astrophysical $S$-factors or rates of various reactions including thermonuclear processes at ultra low energies [1]. Today the known nucleon-nucleon interactions are widely used for studies of $N-d, N-{ }^{3} \mathrm{He}, N-{ }^{3} \mathrm{H}$ and $N-{ }^{4} \mathrm{He}$ processes within different few-body methods. The exact treatment of nuclei starting from the constituent nucleons and the fundamental interactions among them has been a long-standing goal in nuclear physics [2]. In the case of collision of particles composed of identical protons and identical neutrons the effective interactions should be constructed which, in principle, change whenever colliding particles are different or methods of construction are used. However, in general the effective interaction are energy-dependent and complex. The scattering theory allows the calculation of the scattering cross sections as a function of the scattering angle and energy for any potential. In the case of nuclear reactions, the potential of interaction between two colliding particles a priori is unknown. Thus, the task is to get as much information as possible related to the interaction potential through a systematic analysis of the experimental data on the scattering cross section, as well as polarization measurements. The problem of finding the nucleon-nuclear or nuclear-nuclear potential on the basis of the obtained experimental cross sections is twofold: 1) the determination of the phase shifts from obtained experimental scattering cross sections; 2) the construction of the nucleon-nuclear or nuclear-nuclear potential based on the obtained phase shifts of elastic scattering processes [3 [6].

Many nuclear processes of formation of light elements are results of the radiative capture of protons or neutrons by light nuclei as well as collisions of light nuclei. These processes are parts of the nucleosynthesis chain of light elements in the primordial Universe and occur in the early stage of stable star formation [7, 8]. A study of these processes requires a construction of nucleon-nuclear and nuclear-nuclear potentials based on the description of the known scattering phase shifts and the main characteristics of the ground and excited states of nuclei. However, the direct measurement of cross sections of the charged-particle induced reactions at thermonuclear energies becomes almost impossible due to its slump with energy decreasing because of the Coulomb barrier. A common way to get the astrophysical $S$-factors in the low-energy region (stellar energies) is its extrapolation based on the trend of experimental data in the high-energy region [9]. However, prior research [10] demonstrates the danger of simply extrapolating experimental data over a very large energy range towards stellar energies. It also shows the necessity for carefully studying the low-energy reaction cross sections. The predicted light element abundance highly depends on the input data such as the nuclear reaction rates, the equation of state, the opacity of the stellar matter. Nuclear astrophysics reaction rate determines the possibility of a particular reaction, thereby affecting the process of stellar evolution and elements formation. In reaction network calculations, all reactions related to formation and destruction of light nuclei should be taken into account in order to get accurate results [11]. In particular, this related to ${ }^{6} \mathrm{Li}$ and ${ }^{7} \mathrm{Li}$ abundance [6, 12 14]. Therefore, a careful theoretical analysis of astrophysical reactions at low energies requires the corresponding nucleon-nuclear and nuclear-nuclear potentials.

Phase shift analysis is usually performed on the basis of expressions for the elastic scattering cross sections of nuclear particles, namely, on the basis of the representation of the differential cross sections for the elastic scattering through the phase shifts of elastic scattering $15-17$. Many of these expressions can be found in the scientific literature, and were subsequently collected in books [18] and [17]. For example, the scattering of spinless particles on a target with also zero spin and the system of non-identical particles with a channel spin $S=1 / 2$, such as $N-{ }^{4} \mathrm{He}$, is described in detail in the books [5, 17]. However, a particular difficulty is the calculation of differential cross sections for high-spin 
states (channel spin $S>1$ ) of a system of particles. Results for a high channel spin, in particular, for a particle system with the spin structure $1 / 2+1$ and $1 / 2+3 / 2$ a high channel spin were given earlier in Refs. [19] and [20], respectively. However, we still lack fully analytical expressions for calculations of the differential elastic scattering cross sections for half-integer channel spins $S=3 / 2$ and $S=5 / 2$ and integer channel spins $S=1$ and $S=2$. We already reported preliminary calculations related to these channel spins [21]. This article presents the final analytical expressions for calculations of the differential cross sections for the elastic scattering of non-identical nuclear particles. All differential cross sections are expressed in the terms of the orbital quantum number $l$, which allows one to explicitly take into account in the calculations a certain energy-dependent number of scattering partial waves and to consider the relative contribution of each partial wave. These expressions allow one to perform the phase shift analyses based on experimental cross sections obtained for nuclear-nuclear scattering for the integer and half-integer channel spin of the spin-1/2-spin-3/2, spin-1/2- spin-1, and spin-1/2-spin-2 and spin- $1-$ spin-3/2 processes. The amplitudes obtained for particular channel spins, along with the data on elastic channels, make it possible to carry out full phase shift analysis with channel coupling. Analyses and calculations of cross sections and reactions rates at astrophysical energies require a small number of partial waves. In this case from general formulas presented in this work one obtains simple and convenient algebraic expressions for the partial amplitudes.

This work is organized as follows: in Sec. II we present the main ingredients for the description of differential cross sections. Processes with spin- $1 / 2-$ spin-3/2, spin- $1 / 2-$ spin- 1 , and spin- $1 / 2-$ spin- 2 and spin- $1-$ spin-3/2 are considered in Secs. III, IV] and V] respectively. The analytical expressions for the independent partial amplitudes for above mentioned processes are given in Appendixes A, B and C, correspondingly. Conclusions follow in Sec. VI.

\section{THEORETICAL FORMALISM FOR PARTICLES WITH SPINS}

We consider in general a collision of two particles with spins. One of the particles can be a nucleon or the both particles are nuclei. When the two colliding particles have spins, these spins may be reoriented by the scattering even if internal excitations of nuclei do not occur. The total angular momentum and its projection are conserved so that flipping one spin, which is related to the changing the value of its projection on the $z$-axis is compensated by a flip of the other or by an exchange of angular momentum with the relative orbital motion. In the latter case, the plane of the orbits is tilted.

The differential cross section of the elastic scattering channel can be written as [18]

$$
\frac{d \sigma}{d \Omega}=\sum_{S \nu \nu^{\prime}} g(S) \frac{d \sigma(S)}{d \Omega}
$$

where $g(S)$ is the statistical weight for channel spin $S$

$$
g(S)=\frac{2 S+1}{\left(2 s_{1}+1\right)\left(2 s_{2}+1\right)} .
$$

The spin of the scattering reaction channel is the result of the vector addition of the spins $\mathbf{s}_{1}$ and $\mathbf{s}_{2}$ of the incident and target particles and is

$$
\mathbf{S}=\mathbf{s}_{1}+\mathbf{s}_{2}, \quad\left|s_{1}-s_{2}\right| \leq S \leq s_{1}+s_{2} .
$$

In general case the partial differential cross sections can be written as

$$
\frac{d \sigma(S)}{d \Omega}=\sum_{S S^{\prime} \nu \nu^{\prime}} g(S) M_{S^{\prime} \nu^{\prime}}^{S \nu^{*}} M_{S^{\prime} \nu^{\prime}}^{S \nu}
$$

where $M_{S^{\prime} \nu^{\prime}}^{S \nu}$ are the matrix elements of the transition $M$-matrix. Here we assume the general case when the potential couples spins of both collided particles. For the elastic scattering when $S=S^{\prime}$, Eq. (4) describes the partial cross section in Eq. (11). In the channel spin representation, the relationship between the transition $M-$ matrix and the scattering or collision matrix $U$ in the general case, when the initial and final channel spins $S$ and $S^{\prime}$ can take any possible values, is described by the expansion of the scattering amplitude in terms of the Legendre polynomials $P_{n}^{m}(\theta)$ as [22]: 


$$
\begin{aligned}
M_{S^{\prime} \nu^{\prime}}^{S \nu}(\theta)= & f(\theta) \delta_{S S^{\prime}} \delta_{\nu \nu^{\prime}}+\frac{i}{2 k} \sum_{J l l^{\prime}} \sqrt{\frac{\left(l^{\prime}-m^{\prime}\right) !}{\left(l^{\prime}+m^{\prime}\right) !}} C_{S \nu l 0}^{J \nu} C_{S^{\prime} \nu^{\prime} l^{\prime} m^{\prime}}^{J \nu} \\
& \left.\times \exp \left(i\left(\omega_{l}+\omega_{l^{\prime}}\right)\right)\left(\delta_{S S^{\prime}} \delta_{l l^{\prime}}-U_{S^{\prime} l^{\prime} S l}^{J \pi}\right) P_{l^{\prime}}^{m^{\prime}}(\theta)\right] .
\end{aligned}
$$

In Eq. (5) the following notations are used: $S$ and $S^{\prime}$ are the spins of the initial and final channels, respectively, $\nu$ and $\nu^{\prime}$ are their projections on $z$-axis, $l$ and $l^{\prime}$ are the orbital quantum numbers of the initial and final channels, $C_{a \alpha b \beta}^{c \gamma}$ are the Clebsch-Gordan coefficients, $\omega_{l}$ are the Coulomb scattering phase shifts, and $f(\theta)$ and $U_{S^{\prime} l^{\prime} S l}^{J \pi}$ are the Coulomb scattering amplitude and the scattering matrix, respectively. The Coulomb amplitude is defined as follows

$$
f_{c}(\theta)=-\left(\frac{\gamma}{2 k \sin ^{2}(\theta / 2)}\right) \exp \left(i \gamma \ln \left[\sin ^{-2}(\theta / 2)\right]+2 i \omega_{0}\right)
$$

In Eq. (6) $\theta$ is the scattering angle, $k$ is the wave number of the relative motion of collided particles, $k^{2}=\frac{2 \mu E}{\hbar^{2}}$, where $\mu$ is the reduced mass of particles and $E$ is the energy of colliding particles in the center-of-mass system, $\gamma=\frac{\mu Z_{1} Z_{2} e^{2}}{k \hbar^{2}}$ is the Coulomb parameter, where $Z$ is the particle's charge in units of the elementary charge. For a proper description of the scattering cross section, it is sufficient to take into account only elastic channels. Then the collision $U$-matrix takes the form:

$$
U_{S l}=\exp \left(2 i \delta_{S l}\right)
$$

The description of partial differential cross sections requires only those amplitudes for which $S=S^{\prime}$. The matrix elements with $S \neq S^{\prime}$ can be used for calculations of polarization effects, which require the consideration of spin-mixing states. For analytical calculations of elastic scattering cross sections i.e. for the case when the spins of the initial and final channels have the same value $\left(S=S^{\prime}\right)$ it is convenient to present the partial amplitudes of the $M$-matrix in a more succinct form. For this case the expression (5) could be written in the following form

$$
\tilde{M}_{\nu^{\prime}}^{S \nu}(\theta) \equiv M_{S \nu^{\prime}}^{S \nu}(\theta)=\sum_{J l m} C_{S \nu l 0}^{J \nu} C_{S \nu^{\prime} l m}^{J \nu} A_{J l}^{S} Y_{l m}(\theta, 0)
$$

where

$$
A_{J l}^{S}(\theta)=\frac{\sqrt{\pi}}{k} \begin{cases}f_{c}(\theta)+i(2 l+1)^{1 / 2} \exp \left(2 i \omega_{l}\right)\left(1-U_{S l}^{J}\right), & \text { if } \quad \nu=\nu^{\prime} \\ i(2 l+1)^{1 / 2} \exp \left(2 i \omega_{l}\right)\left(1-U_{S l}^{J}\right), & \text { if } \quad \nu \neq \nu^{\prime}\end{cases}
$$

The next step consists in recoupling procedure for the Clebsch-Gordan coefficients in (8) using the relevant expression from [23]

$$
C_{a \alpha f \varphi}^{d \delta} C_{b-\beta e \varepsilon}^{d \delta}=\sum_{c \gamma}(-1)^{c+d-\beta-\varphi}(2 d+1) C_{a \alpha b \beta}^{c \gamma} C_{f-\varphi e \varepsilon}^{c \gamma}\left\{\begin{array}{lll}
a & b & c \\
e & f & d
\end{array}\right\}
$$

where $\left\{\begin{array}{l}\ldots \\ \ldots\end{array}\right\}$ are Wigner $6 j$-symbols. Therefore, using one-to-one correspondence for the $\{a f d\} \rightarrow\{S l J\}$ and $\{b e d\} \rightarrow\{S l J\}$ we can transform the product of the Clebsch - Gordan coefficients as

$$
C_{S \nu l 0}^{J \nu} C_{S \nu^{\prime} l m}^{J \nu}=\sum_{\kappa m}(-1)^{\kappa+J-\nu^{\prime}}(2 J+1) C_{S \nu S-v^{\prime}}^{\kappa m} C_{l 0 l m}^{\kappa m}\left\{\begin{array}{c}
S S \kappa \\
l l J
\end{array}\right\} .
$$

According to the rules of addition of angular momenta, the summation by $\kappa$ in Eq. (11) includes the integers in the intervals $0 \leq \kappa \leq 2 S$ and $0 \leq \kappa \leq 2 l$.

Using the following symmetry properties of the Clebsch-Gordan coefficients [23] 


$$
\begin{aligned}
& C_{a \alpha b \beta}^{c \gamma}=(-1)^{a+b-c} C_{b \beta a \alpha}^{c \gamma}, \\
& C_{a \alpha b \beta}^{c \gamma}=(-1)^{b+\beta} \sqrt{\frac{2 c+1}{2 a+1}} C_{c-\gamma b \beta}^{a-\alpha}, \\
& C_{a \alpha b \beta}^{c \gamma}=(-1)^{a+b-c} C_{a-\alpha b-\beta}^{c-\gamma},
\end{aligned}
$$

the first coefficient $C_{S \nu S-v^{\prime}}^{\kappa m}$ in Eq. (11) can be rewritten as

$$
C_{S \nu S-\nu^{\prime}}^{\kappa m}=(-1)^{S+\kappa+\nu^{\prime}} \sqrt{\frac{2 \kappa+1}{2 S+1}} C_{\kappa m S \nu^{\prime}}^{S \nu}
$$

Thus, Eqs. (9), (11) and (13) allow one to obtain the following form of the matrix elements $\widetilde{M}_{\nu^{\prime}}^{S \nu}(\theta)$ :

$$
\tilde{M}_{\nu^{\prime}}^{S \nu}(\theta)=\sum_{\kappa J l m}(-1)^{J+S}(2 J+1) \sqrt{\frac{2 \kappa+1}{2 S+1}} C_{\kappa m S \nu^{\prime}}^{S \nu} C_{l 0 l m}^{\kappa m}\left\{\begin{array}{c}
S S \kappa \\
l l J
\end{array}\right\} A_{J l}^{S} Y_{l m}(\theta, 0)
$$

Let us turn now to the construction of the differential cross section basing on the $\tilde{M}_{\nu^{\prime}}^{S \nu}$ matrix elements. According to (1) the differential cross section can be written as

$$
\frac{d \sigma(S)}{d \Omega}=\sum_{S \nu \nu^{\prime}} g(S) \tilde{M}_{\nu^{\prime}}^{S \nu^{*}} \tilde{M}_{\nu^{\prime}}^{S \nu}
$$

Substituting Eq. (14) into (15) we can perform the external summation over $\nu \nu^{\prime}$ using the well-known relation for the Clebsch-Gordan coefficients [23]

$$
\sum_{a \alpha} C_{a \alpha b \beta}^{c \gamma} C_{a \alpha b \beta^{\prime}}^{c \gamma^{\prime}}=\frac{\Pi_{c}^{2}}{\Pi_{b}^{2}} \delta_{b b^{\prime}} \delta_{\beta \beta^{\prime}}, \text { where } \Pi_{a b \ldots c}=\sqrt{(2 a+1)(2 b+1) \ldots(2 c+1)} .
$$

The application of (16) leads to

$$
\sum_{v v^{\prime}} C_{\kappa m S \nu^{\prime}}^{S \nu} C_{\kappa^{\prime} m^{\prime} S \nu^{\prime}}^{S \nu}=\delta_{\kappa \kappa^{\prime}} \delta_{m m^{\prime}} \frac{2 S+1}{2 \kappa+1}
$$

Thus, considering (14) and (17) in Eq. (15), we obtain the final expression for the differential cross section as follows:

$$
\frac{d \sigma}{d \Omega}=\sum_{S \kappa} g(S)\left|\sum_{J l m}(-1)^{J}(2 J+1) C_{l 0 l m}^{\kappa m}\left\{\begin{array}{c}
S S \kappa \\
l l J
\end{array}\right\} A_{J l}^{S} Y_{l m}(\theta, 0)\right|^{2}
$$

It should be noted that this expression for the differential cross section has a universal form and can be applied to any processes of elastic scattering, regardless of whether the channel spins are integer or half-integer.

In Table $\Pi$ are listed the light nuclei with the spins $1 / 2,1,3 / 2$ and 2 relevant to the nucleosynthesis chain of light elements in the primordial Universe.

\section{PROCESSES WITH SPIN- $1 / 2-$ SPIN-3/2}

Let us consider the spin states for the collision of two particles with spin $1 / 2$ and $3 / 2$. In this case the collision of following particles from Table \ can be considered: $n, p,{ }^{3} \mathrm{H}\left(1 / 2^{+}\right),{ }^{3} \mathrm{He}\left(1 / 2^{+}\right)$as the first particle and ${ }^{5} \mathrm{Li}\left(3 / 2^{-}\right)$, 
${ }^{7} \mathrm{Li}\left(3 / 2^{-}\right),{ }^{9} \mathrm{Li}\left(3 / 2^{-}\right),{ }^{7} \mathrm{Be}\left(3 / 2^{-}\right),{ }^{9} \mathrm{Be}\left(3 / 2^{-}\right),{ }^{7} \mathrm{~B}\left(3 / 2^{-}\right),{ }^{9} \mathrm{~B}\left(3 / 2^{-}\right),{ }^{11} \mathrm{~B}\left(3 / 2^{-}\right),{ }^{13} \mathrm{~B}\left(3 / 22^{-}\right),{ }^{15} \mathrm{~B}\left(3 / 2^{-}\right),{ }^{9} \mathrm{C}\left(3 / 2^{-}\right)$, ${ }^{11} \mathrm{C}\left(3 / 2^{-}\right),{ }^{17} \mathrm{C}\left(3 / 2^{+}\right),{ }^{13} \mathrm{O}\left(3 / 2^{-}\right)$as the second one.

In this case channel spin can be $S=1$ and $S=2$ according to the vector addition (3). For the channel with the integer spin values 1 and 2 , the $M$-matrix is represented as

$$
\begin{aligned}
& \begin{array}{lllllllll}
S v & 22 & 21 & 20 & 2-1 & 2-2 & 11 & 10 & 1-1
\end{array} \\
& S^{\prime} v^{\prime}
\end{aligned}
$$

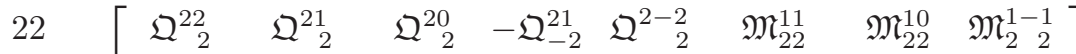

$$
\begin{aligned}
& 21 \quad \begin{array}{llllllll}
\mathfrak{Q}_{1}^{22} & \mathfrak{Q}^{21} & \mathfrak{Q}_{1}^{20} & \mathfrak{Q}^{2-1} & -\mathfrak{Q}_{-1}^{22} & \mathfrak{M}_{21}^{11} & \mathfrak{M}_{21}^{10} & \mathfrak{M}_{2}^{1-1}
\end{array}
\end{aligned}
$$

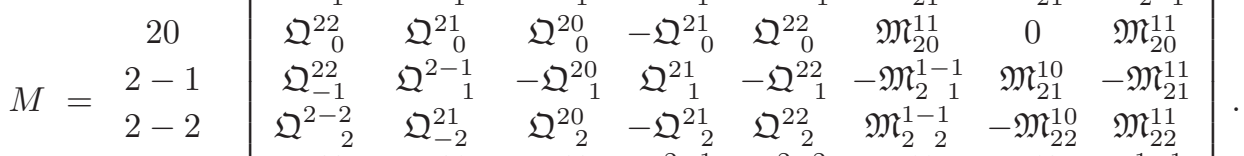

$$
\begin{aligned}
& 11 \quad \begin{array}{llllllll}
\mathfrak{M}_{11}^{22} & \mathfrak{M}_{11}^{21} & \mathfrak{M}_{11}^{20} & \mathfrak{M}_{1}^{2-1} & \mathfrak{M}_{1}^{2-2} & \mathcal{T}_{1}^{11} & \mathcal{T}_{1}^{10} & \mathcal{T}^{1-1}
\end{array} \\
& \begin{array}{l|llllllll}
\mathfrak{M}_{10}^{22} & \mathfrak{M}_{10}^{21} & 0 & \mathfrak{M}_{10}^{21} & -\mathfrak{M}_{10}^{22} & \mathcal{T}_{0}^{11} & \mathcal{T}_{0}^{10} & -\mathcal{T}_{0}^{11}
\end{array} \\
& 1-1 \quad\left[\begin{array}{llllllll}
\mathfrak{M}_{1}^{2-2} & -\mathfrak{M}_{1}^{2-1} & \mathfrak{M}_{11}^{20} & -\mathfrak{M}_{11}^{21} & \mathfrak{M}_{11}^{22} & \mathcal{T}_{1}^{1-1} & -\mathcal{T}_{1}^{10} & \mathcal{T}_{1}^{11}
\end{array}\right]
\end{aligned}
$$

In matrix (19) we use the calligraphic letter $\mathcal{T}$ for the notation of triplet spin states for partial amplitudes and the Fraktur letters $\mathfrak{Q}$ and $\mathfrak{M}$ have been denoted for the quintet and spin-mixing states partial amplitudes, respectively. We especially labeled spin-mixing states by the letter $\mathfrak{M}$ to avoid later confusion with the general labeling of matrix elements of the $M$-matrix.

The matrix (19) has in total 64 partial amplitudes. According to the parity conservation which restricts the sum $l+l^{\prime}$ to being even, it is easy to show that

$$
M_{S^{\prime}-v^{\prime}}^{S-v}=\left\{\begin{array}{lll}
(-1)^{S^{\prime}-S+v^{\prime}-v} M_{S^{\prime} v^{\prime}}^{S v}, & \text { if } & S \neq S^{\prime} \\
(-1)^{v^{\prime}-v} \tilde{M}_{v^{\prime}}^{S v}, & \text { if } & S=S^{\prime}
\end{array}\right.
$$

Thus, it is possible to reduce the number of independent partial amplitudes from 64 to 34 . Considering that 2 of 16 spin-mixing partial amplitudes equal 0 , finally 32 independent $M$-matrix elements remain.

The differential cross section for the elastic scattering in a system of two particles with spin-1/2- spin-3/2, taking into account the spin-orbit interaction, is presented in the form:

$$
\frac{d \sigma(\theta)}{d \Omega}=\frac{3}{8} \frac{d \sigma_{\mathcal{T}}}{d \Omega}+\frac{5}{8} \frac{d \sigma_{\mathfrak{Q}}}{d \Omega} .
$$

The differential cross section $d \sigma_{\mathcal{T}} / d \Omega$ corresponds to the triplet state with the channel spin $S=S^{\prime}=1$ and can be constructed as a combination of independent partial amplitudes from the matrix (19). The differential cross section for the elastic scattering in the triplet channel is determined only by five independent partial amplitudes $\mathcal{T}_{0}^{10}$, $\mathcal{T}_{1}^{11}, \mathcal{T}_{1}^{10}, \mathcal{T}_{0}^{11}$, and $\mathcal{T}_{1}^{1-1}$ given by $3 \times 3$ matrix in the lower corner of the matrix (19) and can by written as

$$
\frac{d \sigma_{\mathcal{T}}}{d \Omega}=\frac{1}{3}\left[\left|\mathcal{T}_{0}^{10}\right|^{2}+2\left(\left|\mathcal{T}_{1}^{11}\right|^{2}+\left|\mathcal{T}_{1}^{10}\right|^{2}+\left|\mathcal{T}_{0}^{11}\right|^{2}+\left|\mathcal{T}_{1}^{1-1}\right|^{2}\right)\right]
$$

TABLE I: Possible incident particles and targets. $J^{\pi}$ is the angular momentum and parity.

\begin{tabular}{cccccccccccccccccc}
\hline$Z$ & 0 & 1 & 1 & 1 & 1 & 2 & 3 & 3 & 3 & 3 & 3 & 3 & 3 & 4 & 4 & 4 & 5 \\
\hline Particle & $n$ & $p$ & $\mathrm{~d}$ & ${ }^{3} \mathrm{H}$ & ${ }^{4} \mathrm{H}$ & ${ }^{3} \mathrm{He}$ & ${ }^{3} \mathrm{Li}$ & ${ }^{4} \mathrm{Li}$ & ${ }^{5} \mathrm{Li}$ & ${ }^{6} \mathrm{Li}$ & ${ }^{7} \mathrm{Li}$ & ${ }^{9} \mathrm{Li}$ & ${ }^{10} \mathrm{Li}$ & ${ }^{7} \mathrm{Be}$ & ${ }^{9} \mathrm{Be}$ & ${ }^{11} \mathrm{Be}$ & ${ }^{7} \mathrm{~B}$ \\
\hline$J^{\pi}$ & $1 / 2^{+}$ & $1 / 2^{+}$ & $1^{+}$ & $1 / 2^{+}$ & $2^{-}$ & $1 / 2^{+}$ & $2^{+}$ & $2^{-}$ & $3 / 2^{-}$ & $1^{+}$ & $3 / 2^{-}$ & $3 / 2^{-}$ & $1^{-}$ & $3 / 2^{-}$ & $3 / 2^{-}$ & $1 / 2^{+}$ & $3 / 2^{-}$ \\
\hline \hline$Z$ & 5 & 5 & 5 & 5 & 5 & 5 & 5 & 6 & 6 & 6 & 6 & 6 & 6 & 7 & 7 & 7 & 7 \\
\hline Particle & ${ }^{8} \mathrm{~B}$ & ${ }^{9} \mathrm{~B}$ & ${ }^{11} \mathrm{~B}$ & ${ }^{12} \mathrm{~B}$ & ${ }^{14} \mathrm{~B}$ & ${ }^{15} \mathrm{~B}$ & ${ }^{17} \mathrm{~B}$ & ${ }^{9} \mathrm{C}$ & ${ }^{11} \mathrm{C}$ & ${ }^{13} \mathrm{C}$ & ${ }^{15} \mathrm{C}$ & ${ }^{17} \mathrm{C}$ & ${ }^{19} \mathrm{C}$ & ${ }^{10} \mathrm{~N}$ & ${ }^{13} \mathrm{~N}$ & ${ }^{15} \mathrm{~N}$ & ${ }^{16} \mathrm{~N}$ \\
\hline$J^{\pi}$ & $2^{+}$ & $3 / 2^{-}$ & $3 / 2^{-}$ & $1^{+}$ & $3 / 2^{-}$ & $2^{-}$ & $3 / 2^{-}$ & $3 / 2^{-}$ & $3 / 2^{-}$ & $1 / 2^{-}$ & $1 / 2^{+}$ & $3 / 2^{+}$ & $1 / 2^{+}$ & $2^{-}$ & $1 / 2^{-}$ & $1 / 2^{-}$ & $2^{-}$ \\
\hline
\end{tabular}


The analytical expressions obtained for the independent partial amplitudes $\mathcal{T}_{v^{\prime}}^{1 v}$, for the spin triplet state are presented in Appendix A by Eqs. A1 - A5.

To describe a quintet spin states with the channel spin $S=S^{\prime}=213$ independent amplitudes are required. The differential cross section $d \sigma_{\mathfrak{Q}} / d \Omega$ for the elastic scattering for the quintet spin state is described by the expression

$$
\begin{aligned}
\frac{d \sigma_{\mathfrak{Q}}}{d \Omega}= & \frac{1}{3}\left[\left|\mathfrak{Q}_{0}^{20}\right|^{2}+2\left(\left|\mathfrak{Q}_{1}^{21}\right|^{2}+\left|\mathfrak{Q}_{2}^{22}\right|^{2}+\left|\mathfrak{Q}_{1}^{20}\right|^{2}+\left|\mathfrak{Q}_{0}^{21}\right|^{2}+\left|\mathfrak{Q}_{1}^{22}\right|^{2}+\left|\mathfrak{Q}_{2}^{21}\right|^{2}+\left|\mathfrak{Q}^{2-1}\right|^{2}+\right.\right. \\
& \left.\left.+\left|\mathfrak{Q}_{2}^{20}\right|^{2}+\left|\mathfrak{Q}_{0}^{22}\right|^{2}+\left|\mathfrak{Q}_{-2}^{21}\right|^{2}+\left|\mathfrak{Q}_{-1}^{22}\right|^{2}+\left|\mathfrak{Q}^{2-2}\right|^{2}\right)\right] .
\end{aligned}
$$

The independent partial amplitudes $\mathfrak{Q}_{0}^{20}, \mathfrak{Q}_{1}^{21}, \mathfrak{Q}_{2}^{22}, \mathfrak{Q}_{1}^{20}, \mathfrak{Q}^{21}, \mathfrak{Q}^{22}, \mathfrak{Q}^{21}, \mathfrak{Q}^{2-1}, \mathfrak{Q}^{20}, \mathfrak{Q}^{22}, \mathfrak{Q}_{-2}^{21}, \mathfrak{Q}_{-1}^{22}$, and $\mathfrak{Q}^{2-2}$ correspond to the case when the total spin in the incoming and outgoing channels are equal and. We obtained the analytical expressions for $\mathfrak{Q}^{2 v} v^{\prime}$ independent partial amplitudes and corresponding formula are given in Appendix A by Eqs. A6 - A18). It should be mentioned that 13 independent amplitudes are listed as the elements of $5 \times 5$ diagonal matrix in the upper corner of the matrix (19).

There are 30 amplitudes $\mathfrak{M}_{2 v^{\prime}}^{1 v}$ and $\mathfrak{M}_{1 v^{\prime}}^{2 v}$ for the spin-mixing states for the collision of particles with the spins $1 / 2$ and $3 / 2$. The number of these amplitudes can be reduced to 14 due to parity conservation and considering that 2 spin-mixing partial amplitudes equal 0 . We obtained the analytical expressions for 7 independent $\mathfrak{M}_{2 v^{\prime}}^{1 v}$ and 7 independent $\mathfrak{M}_{1 v^{\prime}}^{2 v}$ partial spin-mixing amplitudes. The results of calculations of the corresponding amplitudes are given in Appendix A3 by Eqs. A19) - A32).

\section{PROCESSES WITH SPIN-1/2 - SPIN-1}

In this case according to the (3) channel spin can be $S=1 / 2$ and $S=3 / 2$. For the channel with half-integer spin values $1 / 2$ and $3 / 2$ the $M$-matrix is represented as

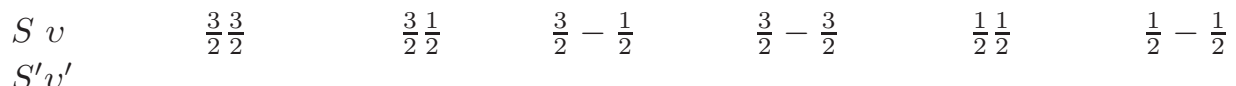

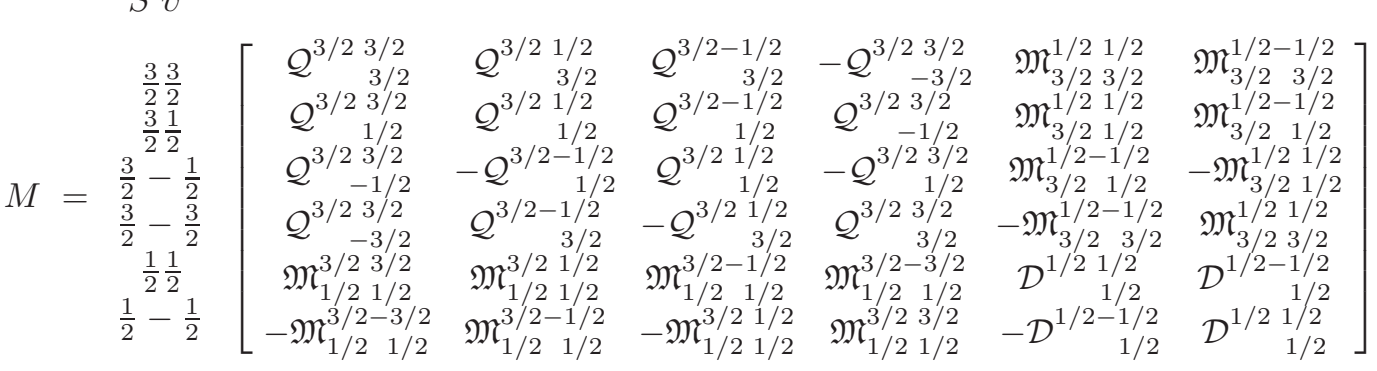

In matrix (24) as above we use as the Fraktur letter $\mathfrak{M}$ for spin-mixing states, while the calligraphic letters $\mathcal{D}$ and $\mathcal{Q}$ have been denoted for the doublet and quintet spin states. The matrix (24) is defined by 36 partial amplitudes. However, according to (20) the number of partial amplitudes of this $M$-matrix can be reduced from 36 to 18 independent partial amplitudes.

The differential cross section for elastic scattering in a system of two particles with spin- $1 / 2-$ spin- 1 , taking into account the spin-orbit interaction, is presented in the form:

$$
\frac{d \sigma(\theta)}{d \Omega}=\frac{1}{3} \frac{d \sigma_{\mathcal{D}}}{d \Omega}+\frac{2}{3} \frac{d \sigma_{\mathcal{Q}}}{d \Omega}
$$

The channel spin $S$ can take values $1 / 2$ (doublet) and $3 / 2$ (quartet). In this case according to Table $n, p,{ }^{3} \mathrm{H}\left(1 / 2^{+}\right)$, ${ }^{3} \mathrm{He}\left(1 / 2^{+}\right)$and ${ }^{2} \mathrm{H}\left(1^{+}\right),{ }^{6} \mathrm{Li}\left(1^{+}\right),{ }^{10} \mathrm{Li}\left(1^{-}\right),{ }^{12} \mathrm{~B}\left(1^{+}\right)$can be considered as colliding particles.

The doublet state, which corresponds to the channel spin $S=S^{\prime}=1 / 2$, is described by two independent amplitudes $\mathcal{D}_{1 / 2}^{1 / 2} \frac{1 / 2}{1 / 2}$ and $\mathcal{D}_{1 / 2}^{1 / 2-1 / 2}$ of the matrix (24), which are presented by $2 \times 2$ matrix in (24). The differential cross section for the elastic scattering in the doublet channel is well known and can be written as

$$
\frac{d \sigma_{\mathcal{D}}}{d \Omega}=\left|\begin{array}{rr}
1 / 2 & 1 / 2 \\
1 / 2
\end{array}\right|^{2}+\left|\begin{array}{r}
1 / 2-1 / 2 \\
1 / 2
\end{array}\right|^{2}
$$


The cross section for the doublet spin channel is defined by two independent partial amplitudes. In Eq. (26) the expressions for the independent partial amplitudes $\mathcal{D}_{1 / 2}^{1 / 2}{ }_{1 / 2}$ and $\mathcal{D}_{1 / 2}^{1 / 2-1 / 2}$ are given by Eqs. (B1) - (B2) in Appendix B.

The description of the quartet spin channel, $S=S^{\prime}=3 / 2$, there are 16 partial amplitudes. However, as it follows from (20), there are only 8 independent $\mathcal{Q}^{3 / 2} v$, amplitudes of the matrix (24) you need to describe the quartet spin channel. The differential cross section for the elastic scattering for a quartet state is defined as

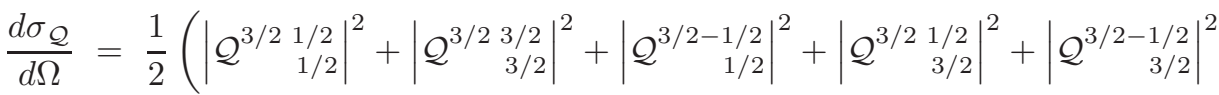

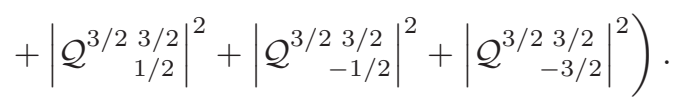

The analytical expressions for the independent partial quartet amplitudes $\mathcal{Q}_{1 / 2}^{3 / 2}{ }_{1 / 2}^{1 / 2}, \mathcal{Q}_{3 / 2}^{3 / 2}, \mathcal{Q}_{1 / 2}^{3 / 2-1 / 2}, \mathcal{Q}_{3 / 2}^{3 / 2}{ }_{3 / 2}^{1 / 2}$, $\mathcal{Q}_{3 / 2}^{3 / 2-1 / 2}, \mathcal{Q}_{1 / 2}^{3 / 2}{ }_{1 / 2}, \mathcal{Q}^{3 / 2}{ }_{-1 / 2}^{3 / 2}$, and $\mathcal{Q}^{3 / 2}{ }_{-3 / 2}^{3 / 2}$ in Eq. (24) are calculated and presented in Appendix B by Eqs. (B33) (B10).

There are 16 partial amplitudes that determine the spin-mixing states. According to Eq. (20) this number can be reduced to 8 . We obtained the analytical expression for the spin-mixing independent partial $\mathfrak{M}_{3 / 2}^{1 / 2} v^{\prime}$ and $\mathfrak{M}_{1 / 2}^{3 / 2} v^{\prime}$ amplitudes of the $M$-matrix for the half-integer channel spin $S=1 / 2$ and $S=3 / 2$. The corresponding amplitudes are presented in Appendix B.3 by Eqs. (B11) - (B18).

\section{PROCESSES WITH SPIN-1/2 - SPIN-2 AND SPIN-1 - SPIN-3/2}

For the channel with the spin value $S=S^{\prime}=5 / 2$ of the incoming and outgoing channel the part of $M$-matrix corresponding to the sextet spin channel is represented as

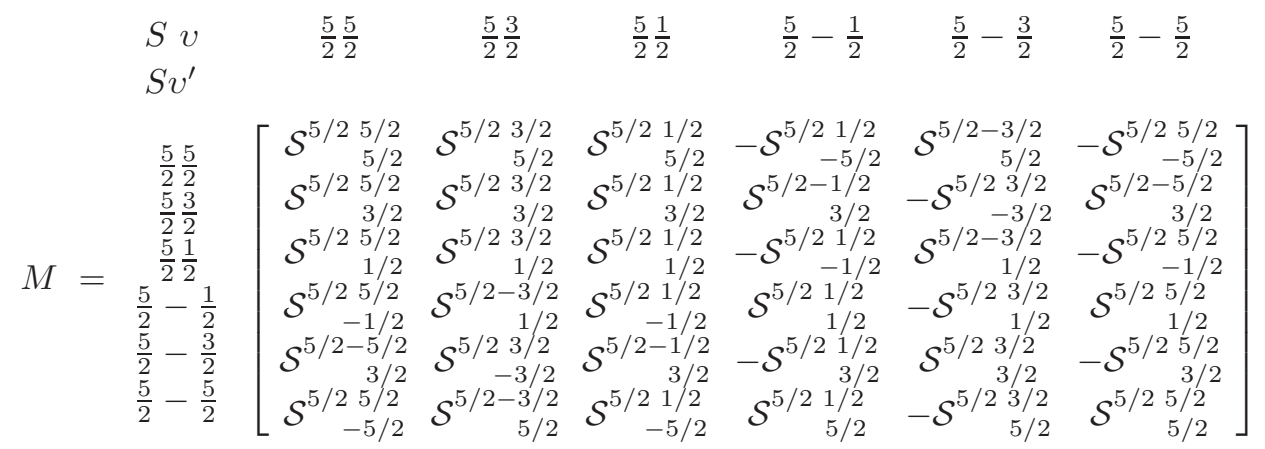

In the interaction of particles with the spins $1 / 2$ and 2 , the channel spin can take the values $S=3 / 2$ (quartet state, $d \sigma_{\mathcal{Q}} / d \Omega$ ) and $S=5 / 2$ (sextet state, $d \sigma_{\mathcal{S}} / d \Omega$ ). In this case the following particles from Table $\prod$ which give the quartet and sextet channel spin can be involved in the collision process: $n, p,{ }^{3} \mathrm{H}\left(1 / 2^{+}\right),{ }^{3} \mathrm{He}\left(1 / 2^{+}\right)$and ${ }^{4} \mathrm{H}\left(2^{-}\right),{ }^{3} \mathrm{Li}\left(2^{+}\right)$, ${ }^{4} \mathrm{Li}\left(2^{-}\right){ }^{8} \mathrm{~B}\left(2^{+}\right),{ }^{14} \mathrm{~B}\left(2^{-}\right),{ }^{10} \mathrm{~N}\left(2^{-}\right),{ }^{16} \mathrm{~N}\left(2^{-}\right)$. The corresponding differential cross section for the elastic scattering is

$$
\frac{d \sigma(\theta)}{d \Omega}=\frac{2}{5} \frac{d \sigma_{\mathcal{Q}}}{d \Omega}+\frac{3}{5} \frac{d \sigma_{\mathcal{S}}}{d \Omega} .
$$

The partial differential cross section for the channel's spin $S=5 / 2$ is represented by the following combination of amplitudes of the matrix (28)

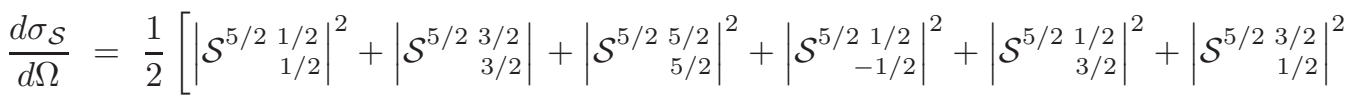

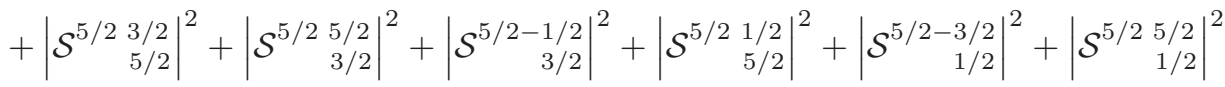

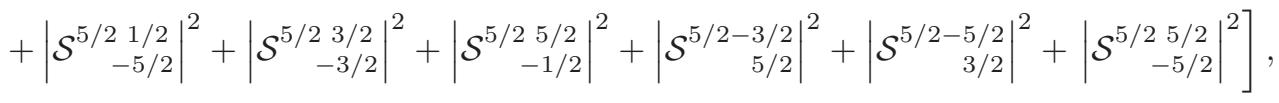


where $\mathcal{S}^{5 / 2} v$, are 18 independent partial amplitudes and the corresponding analytical expressions for $\mathcal{S}^{5 / 2} 1 / 2$,

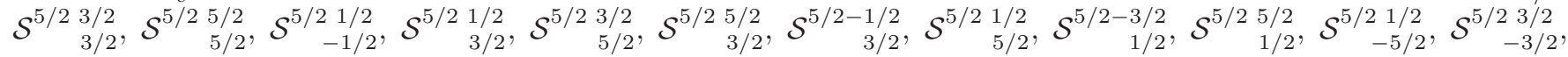
$\mathcal{S}_{-1 / 2}^{5 / 2}, \mathcal{S}_{5 / 2}^{5 / 2-3 / 2}, \mathcal{S}_{3 / 2}^{5 / 2-5 / 2}$, and $\mathcal{S}_{-5 / 2}^{5 / 2}$ amplitudes. Let us emphasize that we labeled the sextet spin channel partial amplitudes by the calligraphic letter $\mathcal{S}$. Results of our calculations for 18 independent partial amplitudes $\mathcal{S}^{5 / 2} v v^{\prime}$ are presented by Eqs. C1 - C18 in Appendix C.

For the system with spin-1/2-spin-2 channel spin $S$ and $S^{\prime}$ can equals $1 / 2,3 / 2$ and $5 / 2$. In this case, the differential cross section for elastic scattering is determined by the expression

$$
\frac{d \sigma(\theta)}{d \Omega}=\frac{1}{6} \frac{d \sigma_{\mathcal{D}}}{d \Omega}+\frac{1}{3} \frac{d \sigma_{\mathcal{Q}}}{d \Omega}+\frac{1}{2} \frac{d \sigma_{\mathcal{S}}}{d \Omega}
$$

The collision of particles $n, p,{ }^{3} \mathrm{H}\left(1 / 2^{+}\right),{ }^{3} \mathrm{He}\left(1 / 2^{+}\right)$and ${ }^{4} \mathrm{H}\left(2^{-}\right),{ }^{3} \mathrm{Li}\left(2^{+}\right),{ }^{4} \mathrm{Li}\left(2^{-}\right){ }^{8} \mathrm{~B}\left(2^{+}\right),{ }^{14} \mathrm{~B}\left(2^{-}\right),{ }^{10} \mathrm{~N}\left(2^{-}\right),{ }^{16} \mathrm{~N}\left(2^{-}\right)$ from Table I. which leads to the channel spin $1 / 2,3 / 2$ and $5 / 2$, can be considered. The corresponding independent partial amplitudes for $\frac{d \sigma_{\mathcal{D}}}{d \Omega}, \frac{d \sigma_{\mathcal{Q}}}{d \Omega}$, and $\frac{d \sigma_{\mathcal{S}}}{d \Omega}$ cross sections are given in Appendixes B and C by Eqs. (C1) - C18) and (C1) - (C18). The general form of the $12 \times 12$ matrix for the processes with spin- $1 / 2-$ spin- 2 and spin- $1-$ spin- $3 / 2$ is presented in Ref. [21]. The number of independent matrix elements can be reduced from 144 to 72 . The doublet spin state with the cannel spin $S=1 / 2$ is described by only 2 partial amplitudes, while the discription of the quartet channel with spin $S=3 / 2$ and the sextet spin state with $S=5 / 2$ are required 8 and 18 independent matrix elements, respectively. This matrix also contains 8 independent spin-mixing amplitudes for the mixing doublet and quartet states. The analytical expressions for all these independent partial amplitudes are presented in Appendixes. However, in the general case of the $12 \times 12$ matrix there are also 12 independent spin-mixing amplitudes $\mathfrak{M}_{5 / 2 v^{\prime}}^{1 / 2 v}$ and $\mathfrak{M}_{1 / 2 v^{\prime}}^{5 / 2 v}$ for the mixing doublet and sextet states, and 24 independent spin-mixing amplitudes $\mathfrak{M}_{5 / 2 v^{\prime}}^{3 / 2 v}$ and $\mathfrak{M}_{3 / 2 v^{\prime}}^{5 / 2 v}$ for the mixing quartet and sextet states. The spin-mixing amplitudes for the latter two cases can be obtained.

\section{CONCLUSIONS}

We present the analytical expressions for the differential cross sections for elastic scattering of nuclear particles for channels with a spin value of $1 / 2,1,3 / 2,2$ and 5/2. The corresponding independent partial amplitudes for each channel spin are obtained. These expressions are presented for arbitrary orbital angular momentum $l$ and taking into account spin-orbit splitting. To describe the triplet state with the channel spin $S=1,5$ independent amplitudes $\mathcal{T}_{v^{\prime}}^{1 v}$ are required. To describe the quintet $(S=2)$, the number of independent amplitudes $\mathfrak{Q}^{2 v} v^{\prime}$ increases to 13 . The number of independent spin-mixing amplitudes $\mathfrak{M}_{2}^{1} v_{v^{\prime}}^{v}$ and $\mathfrak{M}_{1}^{2} v_{v^{\prime}}^{v}$ in this case is 14 . For the description of the half-integer doublet $(S=1 / 2)$ channel spin state only 2 independent partial amplitudes $\mathcal{D}^{1 / 2} v$ are required, while for the quartet state with the channel spin $S=3 / 2$ the number of independent amplitudes $\mathcal{Q}^{3 / 2} v v^{\prime}$ equals 8 . There are 18 independent partial amplitudes $\mathcal{S}_{v^{\prime}}^{5 / 2}$ for the sextet state with the channel spin $S=5 / 2$. In the case of mixing doublet and quartet states 8 independent spin-mixing amplitudes $\mathfrak{M}_{3 / 2}^{1 / 2} v^{\prime}$ and $\mathfrak{M}_{1 / 2}^{3 / 2} v^{\prime}$ are required. Obviously, with an increase of the channel spin, the number of required independent amplitudes for a correct description of scattering processes increases. For low energy processes, whose description requires a small number of partial waves, general expressions for the partial amplitudes can be reduced to simple algebraic expressions.

Using experimental data for a nucleon-nucleus and nuclear-nuclear reaction cross sections the obtained expressions for the differential cross sections and independent partial amplitudes allow perform the phase shifts analyses for different integer and half-integer channel spins, and find corresponding phase shifts. These phase shifts will be used in the most essential stages of construction of a nucleon-nuclear or nucleon-nucleon potentials, which will be further employed for calculations of main characteristics of nuclear reactions, in particular, astrophysical reactions at low energies.

\section{ACKNOWLEDGEMENT}

A. S. Tkachenko acknowledges the support by al-Farabi Kazakh National University, the Physics Department and the Center for Theoretical Physics of New York City College of Technology, CUNY. 


\section{Appendix A: Partial amplitudes for the spin-1-spin-3/2 system}

\section{Triplet spin state}

The independent partial amplitudes that determine the cross section for the scattering in triplet state are:

$$
\begin{gathered}
\mathcal{T}_{0}^{10}=f_{c}(\theta)+\frac{1}{2 i k} \sum_{l=0}\left[(l+1) \alpha^{l+1}+l \alpha^{l-1}\right] \exp \left(2 i \omega_{l}\right) P_{l}(\cos \theta) \\
\mathcal{T}_{1}^{11}=f_{c}(\theta)+\frac{1}{4 i k} \sum_{l=0}\left[(l+2) \alpha^{l+1}+(2 l+1) \alpha^{l}+(l-1) \alpha^{l-1}\right] \exp \left(2 i \omega_{l}\right) P_{l}(\cos \theta), \\
\mathcal{T}_{1}^{10}=\frac{1}{2 \sqrt{2} i k} \sum_{l=1}\left[\alpha^{l+1}-\alpha^{l-1}\right] \exp \left(2 i \omega_{l}\right) P_{l}^{1}(\cos \theta), \\
\mathcal{T}_{0}^{11}=\frac{1}{2 \sqrt{2} i k} \sum_{l=1} \frac{1}{l(l+1)}\left[l(l+2) \alpha^{l+1}-(2 l+1) \alpha^{l}-(l-1)(l+1) \alpha^{l-1}\right] \exp \left(2 i \omega_{l}\right) P_{l}^{1}(\cos \theta), \\
\mathcal{T}_{1}^{1-1}=\frac{1}{4 i k} \sum_{l=2} \frac{1}{l(l+1)}\left[\alpha^{l+1}-(2 l+1) \alpha^{l}+(l+1) \alpha^{l-1}\right] \exp \left(2 i \omega_{l}\right) P_{l}^{2}(\cos \theta) .
\end{gathered}
$$

The value $\alpha^{J}=\left(U_{s l}^{J^{\pi}}-1\right)$ for each state with full momentum $J$ is entered here.

\section{Quintet spin state}

The independent partial amplitudes that determine the cross section for the scattering in quintet state are:

$$
\begin{aligned}
& \mathfrak{Q}_{0}^{20}= f_{c}(\theta)+\frac{1}{4 i k} \sum_{l=0}\left[\frac{3(l+1)(l+2)}{2 l+3} \alpha^{l+2}+\frac{2 l(l+1)(2 l+1)}{(2 l+3)(2 l-1)} \alpha^{l}+\frac{3 l(l-1)}{2 l-1} \alpha^{l-2}\right] \\
& \times \exp \left(2 i \omega_{l}\right) P_{l}(\cos \theta), \\
& \mathfrak{Q}^{21}= f_{c}(\theta)+\frac{1}{4 i k} \sum_{l=0}\left[\frac{2(l+1)(l+3)}{2 l+3} \alpha^{l+2}+l \alpha^{l+1}+\frac{3(2 l+1)}{(2 l+3)(2 l-1)} \alpha^{l}+(l+1) \alpha^{l-1}\right. \\
&\left.+\frac{2 l(l-2)}{2 l-1} \alpha^{l-2}\right] \exp \left(2 i \omega_{l}\right) P_{l}(\cos \theta), \\
& \mathfrak{Q}^{22}{ }_{2}= f_{c}(\theta)+\frac{1}{8 i k} \sum_{l=0}\left[\frac{(l+3)(l+4)}{2 l+3} \alpha^{l+2}+2(l+3) \alpha^{l+1}+\frac{6(l+2)(2 l+1)(l-1)}{(2 l-1)(2 l+3)} \alpha^{l}+\right. \\
&\left.2(l-2) \alpha^{l-1}+\frac{(l-2)(l-3)}{2 l-1} \alpha^{l-2}\right] \exp \left(2 i \omega_{l}\right) P_{l}(\cos \theta), \\
& \mathfrak{Q}^{20}{ }_{1}=-\frac{\sqrt{6}}{4 i k} \sum_{l=2} \frac{1}{(2 l+3)(2 l-1)}\left[(l+2)(2 l-1) \alpha^{l+2}-(2 l+1) \alpha^{l}\right. \\
&\left.-(2 l+3)(l-1) \alpha^{l-2}\right] \exp \left(2 i \omega_{l}\right) P_{l}^{2}(\cos \theta),
\end{aligned}
$$




$$
\begin{aligned}
& \mathfrak{Q}_{0}^{21}=-\frac{\sqrt{6}}{4 i k} \sum_{l=1}\left[\frac{l+3}{2 l+3} \alpha^{l+2}-\frac{1}{l+1} \alpha^{l+1}-\frac{(2 l+1)(3-l(l+1))}{l(l+1)(2 l+3)(2 l-1)} \alpha^{l}-\frac{1}{l} \alpha^{l-1}\right. \\
& \left.-\frac{l-2}{2 l-1} \alpha^{l-2}\right] \exp \left(2 i \omega_{l}\right) P_{l}^{1}(\cos \theta) \text {, } \\
& \mathfrak{Q}_{1}^{22}=-\frac{1}{4 i k} \sum_{l=1}\left[\frac{(l+3)(l+4)}{(2 l+3)(l+1)} \alpha^{l+2}+\frac{(l+3)(l-2)}{l(l+1)} \alpha^{l+1}-\frac{9(l+2)(2 l+1)(l-1)}{l(2 l-1)(2 l+3)(l+1)} \alpha^{l}\right. \\
& \left.-\frac{(l+3)(l-2)}{l(l+1)} \alpha^{l-1}-\frac{(l-2)(l-3)}{l(2 l-1)} \alpha^{l-2}\right] \exp \left(2 i \omega_{l}\right) P_{l}^{1}(\cos \theta), \\
& \mathfrak{Q}_{2}^{21}=-\frac{1}{4 i k} \sum_{l=1}\left[\frac{l+3}{2 l+3} \alpha^{l+2}+\alpha^{l+1}-\frac{3(2 l+1)}{(2 l+3)(2 l-1)} \alpha^{l}-\alpha^{l-1}\right. \\
& \left.-\frac{l-2}{2 l-1} \alpha^{l-2}\right] \exp \left(2 i \omega_{l}\right) P_{l}^{1}(\cos \theta) \text {, } \\
& \mathfrak{Q}_{1}^{2-1}=\frac{1}{4 i k} \sum_{l=2}\left[\frac{2(l+3)}{(2 l+3)(l+2)} \alpha^{l+2}-\frac{l+4}{(l+1)(l+2)} \alpha^{l+1}-\frac{9(2 l+1)}{l(2 l-1)(2 l+3)(l+1)} \alpha^{l}\right. \\
& \left.-\frac{l-3}{l(l-1)} \alpha^{l-1}+\frac{l-2}{(2 l-1)(l-1)} \alpha^{l-2}\right] \exp \left(2 i \omega_{l}\right) P_{l}^{2}(\cos \theta) \\
& \mathfrak{Q}_{2}^{20}=-\frac{\sqrt{6}}{8 i k} \sum_{l=2} \frac{1}{(2 l+3)(l-1)}\left[(2 l-1) \alpha^{l+2}-2(2 l+1) \alpha^{l}+(2 l+3) \alpha^{l-2}\right] \exp \left(2 i \omega_{l}\right) P_{l}^{2}(\cos \theta) \\
& \mathfrak{Q}_{0}^{22}=\frac{\sqrt{6}}{8 i k} \sum_{l=2}\left[\frac{(l+3)(l+4)}{(2 l+3)(l+1)(l+2)} \alpha^{l+2}-\frac{4(l+3)}{l(l+1)(l+2)} \alpha^{l+1}-\frac{4(2 l+1)(l+4)(l-1)}{l(l+1)(2 l+3)(2 l-1)} \alpha^{l}\right. \\
& \left.+\frac{4(l-2)}{l(l+1)(l-1)} \alpha^{l-1}+\frac{(l-2)(l-3)}{l(2 l-1)(l-1)} \alpha^{l-2}\right] \exp \left(2 i \omega_{l}\right) P_{l}^{2}(\cos \theta), \\
& \mathfrak{Q}_{-2}^{21}=-\frac{1}{4 i k} \sum_{l=3}\left[\frac{1}{(2 l+3)(l+2)} \alpha^{l+2}-\frac{1}{(l+1)(l+2)} \alpha^{l+1}-\frac{3(2 l+1)}{l(2 l-1)(2 l+3)(l+1)} \alpha^{l}\right. \\
& \left.+\frac{1}{l(l-1)} \alpha^{l-1}-\frac{1}{(2 l-1)(l-1)} \alpha^{l-2}\right] \exp \left(2 i \omega_{l}\right) P_{l}^{3}(\cos \theta) \text {, } \\
& \mathfrak{Q}_{-1}^{22}=-\frac{1}{4 i k} \sum_{l=3}\left[\frac{l+4}{(2 l+3)(l+1)(l+2)} \alpha^{l+2}-\frac{l+6}{l(l+1)(l+2)} \alpha^{l+1}+\frac{15(2 l+1)}{l(2 l-1)(2 l+3)(l+1)} \alpha^{l}\right. \\
& \left.+\frac{l-5}{l(l+1)(l-1)} \alpha^{l-1}-\frac{l-3}{l(2 l-1)(l-1)} \alpha^{l-2}\right] \exp \left(2 i \omega_{l}\right) P_{l}^{3}(\cos \theta) \\
& \mathfrak{Q}_{2}^{2-2}=\frac{1}{8 i k} \sum_{l=4}\left[\frac{1}{(2 l+3)(l+1)(l+2)} \alpha^{l+2}-\frac{2}{l(l+1)(l+2)} \alpha^{l+1}+\frac{6(2 l+1)}{l(2 l-1)(2 l+3)(l+1)} \alpha^{l}\right. \\
& \left.-\frac{2}{l(l+1)(l-1)} \alpha^{l-1}+\frac{1}{l(2 l-1)(l-1)} \alpha^{l-2}\right] \exp \left(2 i \omega_{l}\right) P_{l}^{4}(\cos \theta) \text {. }
\end{aligned}
$$


3. Spin-mixing $M$-matrix elements, integer spin channel values $S=1$ and $S=2$

$$
\begin{aligned}
& \mathfrak{M}_{22}^{11}=\frac{i}{2 k} \sum_{l}\left[\frac{l+2}{\sqrt{8 l(2 l+1)}} \exp \left(i\left(\omega_{l}+\omega_{l-1}\right)\right) U_{2(l-1) 1 l}^{l+1} P_{l-1}^{1}\right. \\
& -\sqrt{\frac{l+2}{4 l}} \exp \left(2 i \omega_{l}\right) U_{2 l 1 l}^{l+1} P_{l}^{1}- \\
& +\sqrt{\frac{(l+2)(l+3)}{2(2 l+1)(2 l+5)}} \exp \left(i\left(\omega_{l}+\omega_{l+1}\right)\right) U_{2(l+1) 1 l}^{l+1} P_{l+1}^{1} \\
& -\sqrt{\frac{l+2}{4(l+3)}} \exp \left(i\left(\omega_{l}+\omega_{l+2}\right)\right) U_{2(l+2) 1 l}^{l+1} P_{l+2}^{1} \\
& +\sqrt{\frac{(l+2)(l+1)}{8(l+3)(2 l+5)}} \exp \left(i\left(\omega_{l}+\omega_{l+3}\right)\right) U_{2(l+3) 1 l}^{l+1} P_{l+3}^{1} \\
& -\sqrt{\frac{(2 l+1)(l+1)}{8(l-1)(2 l-1)}} \exp \left(i\left(\omega_{l}+\omega_{l-2}\right)\right) U_{2(l-2) 1 l}^{l} P_{l-2}^{1} \\
& +\sqrt{\frac{2 l+1}{4(l-1)}} \exp \left(i\left(\omega_{l}+\omega_{l-1}\right)\right) U_{2(l-1) 1 l}^{l} P_{l-1}^{1} \\
& -\sqrt{\frac{2 l+1}{4(2 l-1)(2 l+3)}} \exp \left(2 i \omega_{l}\right) U_{2 l 1 l}^{l} P_{l}^{1} \\
& +\sqrt{\frac{2 l+1}{4(l+2)}} \exp \left(i\left(\omega_{l}+\omega_{l+1}\right)\right) U_{2(l+1) 1 l}^{l} P_{l+1}^{1} \\
& -\sqrt{\frac{l(2 l+1)}{8(l+2)(2 l+3)}} \exp \left(i\left(\omega_{l}+\omega_{l+2}\right)\right) U_{2(l+2) 1 l}^{l} P_{l+2}^{1} \\
& +\sqrt{\frac{l(l-1)}{8(2 l-3)(l-2)}} \exp \left(i\left(\omega_{l}+\omega_{l-3}\right)\right) U_{2(l-3) 1 l}^{l-1} P_{l-3}^{1} \\
& -\sqrt{\frac{l-1}{4(l-2)}} \exp \left(i\left(\omega_{l}+\omega_{l-2}\right)\right) U_{2(l-2) 1 l}^{l-1} P_{l-2}^{1} \\
& +\sqrt{\frac{(l-1)(2 l-1)}{4(2 l-3)(2 l+1)}} \exp \left(i\left(\omega_{l}+\omega_{l-1}\right)\right) U_{2(l-1) 1 l}^{l-1} P_{l-1}^{1} \\
& -\sqrt{\frac{l-1}{4(l+1)}} \exp \left(2 i \omega_{l}\right) U_{2 l 1 l}^{l-1} P_{l}^{1} \\
& \left.+\frac{l-1}{\sqrt{8(l+1)(2 l+1)}} \exp \left(i\left(\omega_{l}+\omega_{l+1}\right)\right) U_{2(l+1) 1 l}^{l-1} P_{l+1}^{1}\right],
\end{aligned}
$$




$$
\begin{aligned}
& \mathfrak{M}_{22}^{10}=\frac{i}{2 k} \sum_{l}\left[\frac{1}{2 \sqrt{l(2 l+1)}} \exp \left(i\left(\omega_{l}+\omega_{l-1^{\prime}}\right)\right) U_{2(l-1) 1 l}^{l+1} P_{l-1}^{2}\right. \\
& -\frac{1}{\sqrt{2 l(l+2)}} \exp \left(2 i \omega_{l}\right) U_{2 l 1 l}^{l+1} P_{l}^{2} \\
& +\sqrt{\frac{3(2 l+3)}{2(2 l+1)(2 l+5)(l+2)}} \exp \left(i\left(\omega_{l}+\omega_{l+1}\right)\right) U_{2(l+1) 1 l}^{l+1} P_{l+1}^{2} \\
& -\frac{1}{\sqrt{2(l+2)(l+3)}} \exp \left(i\left(\omega_{l}+\omega_{l+2}\right)\right) U_{2(l+2) 1 l}^{l+1} P_{l+2}^{2} \\
& +\sqrt{\frac{l+1}{4(l+3)(l+2)(2 l+5)}} \exp \left(i\left(\omega_{l}+\omega_{l+3}\right)\right) U_{2(l+3) 1 l}^{l+1} P_{l+3}^{2} \\
& -\sqrt{\frac{l}{4(2 l-3)(l-1)(l-2)}} \exp \left(i\left(\omega_{l}+\omega_{l-3}\right)\right) U_{2(l-3) 1 l}^{l-1} P_{l-3}^{2} \\
& +\frac{1}{\sqrt{2(l-1)(l-2)}} \exp \left(i\left(\omega_{l}+\omega_{l-2}\right)\right) U_{2(l-2) 1 l}^{l-1} P_{l-2}^{2} \\
& -\sqrt{\frac{3(2 l-1)}{2(2 l-3)(2 l+1)(l-1)}} \exp \left(i\left(\omega_{l}+\omega_{l-1}\right)\right) U_{2(l-1) 1 l}^{l-1} P_{l-1}^{2} \\
& +\frac{1}{\sqrt{2(l-1)(l+1)}} \exp \left(2 i \omega_{l}\right) U_{2 l 1 l}^{l-1} P_{l}^{2} \\
& \left.+\frac{1}{\sqrt{4(2 l+1)(l+1)}} \exp \left(i\left(\omega_{l}+\omega_{l+1}\right)\right) U_{2(l+1) 1 l}^{l-1} P_{l+1}^{2}\right],
\end{aligned}
$$




$$
\begin{aligned}
& \mathfrak{M}_{2}^{1-1}=\frac{i}{2 k} \sum_{l}\left[\sqrt{\frac{(l+2)(l-2)(l-3)}{8 l(2 l+1)(l+1)}} \exp \left(i\left(\omega_{l}+\omega_{l-1^{\prime}}\right)\right) U_{2(l-1) 1 l}^{l+1} P_{l-1}^{1}\right. \\
& +\frac{\sqrt{(l+2)(l+3)(l-2)(l-1)}}{2 l(l+1)} \exp \left(2 i \omega_{l}\right) U_{2 l 1 l}^{l+1} P_{l}^{1} \\
& +\sqrt{\frac{3 l(l+3)(l+4)(2 l+3)(l-1)}{2(l+1)^{2}(l+2)(2 l+1)(2 l+5)}} \exp \left(i\left(\omega_{l}+\omega_{l+1}\right)\right) U_{2(l+1) 1 l}^{l+1} P_{l+1}^{1} \\
& -\sqrt{\frac{l(l+5)(l+4)}{4(l+1)(l+2)(l+3)}} \exp \left(i\left(\omega_{l}+\omega_{l+2}\right)\right) U_{2(l+2) 1 l}^{l+1} P_{l+2}^{1} \\
& -\sqrt{\frac{(l+5)(l+6)}{8(l+3)(2 l+5)}} \exp \left(i\left(\omega_{l}+\omega_{l+3}\right)\right) U_{2(l+3) 1 l}^{l+1} P_{l+3}^{1} \\
& -\sqrt{\frac{(2 l+1)(l-3)(l-4)}{8 l(l-1)(2 l-1)}} \exp \left(i\left(\omega_{l}+\omega_{l-2}\right)\right) U_{2(l-2) 1 l}^{l} P_{l-2}^{1} \\
& +\frac{\sqrt{(2 l+1)(l+2)(l-2)(l-3)}}{2 l(l-1)} \exp \left(i\left(\omega_{l}+\omega_{l-1}\right)\right) U_{2(l-1) 1 l}^{l} P_{l-1}^{1} \\
& -\sqrt{\frac{2 l+1}{2}} \exp \left(2 i \omega_{l}\right) U_{2 l 1 l}^{l} P_{l}^{1} \\
& +\sqrt{\frac{(2 l+1)(l+3)(l+4)(l-1)}{2 l(l+1)^{2}(l+2)}} \exp \left(i\left(\omega_{l}+\omega_{l+1}\right)\right) U_{2(l+1) 1 l}^{l} P_{l+1}^{1} \\
& -\sqrt{\frac{(2 l+1)(l+4)(l+5)}{8(l+1)(l+2)(2 l+3)}} \exp \left(i\left(\omega_{l}+\omega_{l+2}\right)\right) U_{2(l+2) 1 l}^{l} P_{l+2}^{1} \\
& +\sqrt{\frac{(l-4)(l-5)}{8(2 l-3)(l-2)}} \exp \left(i\left(\omega_{l}+\omega_{l-3}\right)\right) U_{2(l-3) 1 l}^{l-1} P_{l-3}^{1} \\
& +\sqrt{\frac{(l+1)(l-3)(l-4)}{4(l-2)^{2}(l-1)}} \exp \left(i\left(\omega_{l}+\omega_{l-2}\right)\right) U_{2(l-2) 1 l}^{l-1} P_{l-2}^{1} \\
& +\sqrt{\frac{3(l-1)(l-2)(l-3)(2 l-1)(l+1)(l+2)}{4(2 l-3)(2 l+1)}} \exp \left(i\left(\omega_{l}+\omega_{l-1}\right)\right) U_{2(l-1) 1 l}^{l-1} P_{l-1}^{1} \\
& -\sqrt{\frac{(l+3)(l+2)(l-2)}{4 l^{2}(l+1)}} \exp \left(2 i \omega_{l}\right) U_{2 l 1 l}^{l-1} P_{l}^{1} \\
& \left.+\sqrt{\frac{(l+3)(l+4)(l-1)}{8 l(l+1)(2 l+1)}} \exp \left(i\left(\omega_{l}+\omega_{l+1}\right)\right) U_{2(l+1) 1 l}^{l-1} P_{l+1}^{1}\right]
\end{aligned}
$$




$$
\begin{aligned}
& \mathfrak{M}_{21}^{11}=\frac{i}{2 k} \sum_{l}\left[\frac{l+2}{\sqrt{2(2 l+1)(l-1)}} \exp \left(i\left(\omega_{l}+\omega_{l-1^{\prime}}\right)\right) U_{2(l-1) 1 l}^{l+1} P_{l-1}^{1}\right. \\
& -\sqrt{\frac{(l+2)}{4(l+1)}} \exp \left(2 i \omega_{l}\right) U_{2 l 1 l}^{l+1} P_{l}^{1} \\
& -\sqrt{\frac{3(2 l+3)}{4(l+1)(2 l+1)(2 l+5)}} \exp \left(i\left(\omega_{l}+\omega_{l+1}\right)\right) U_{2(l+1) 1 l}^{l+1} P_{l+1}^{1} \\
& +\frac{1}{2} \exp \left(i\left(\omega_{l}+\omega_{l+2}\right)\right) U_{2(l+2) 1 l}^{l+1} P_{l+2}^{1} \\
& -\sqrt{\frac{(l+2)(l+1)}{2(l+4)(2 l+5)}} \exp \left(i\left(\omega_{l}+\omega_{l+3}\right)\right) U_{2(l+3) 1 l}^{l+1} P_{l+3}^{1} \\
& -\sqrt{\frac{(2 l+1)(l+1)}{2(l-2)(2 l-1)}} \exp \left(i\left(\omega_{l}+\omega_{l-2}\right)\right) U_{2(l-2) 1 l}^{l} P_{l-2}^{1} \\
& +\sqrt{\frac{2 l+1}{4 l}} \exp \left(i\left(\omega_{l}+\omega_{l-1}\right)\right) U_{2(l-1) 1 l}^{l} P_{l-1}^{1} \\
& +\frac{\sqrt{3}(2 l+1)}{\sqrt{4 l(2 l-1)(2 l+3)(l+1)}} \exp \left(2 i \omega_{l}\right) U_{2 l 1 l}^{l} P_{l}^{1} \\
& -\sqrt{\frac{2 l+1}{4(l+1)}} \exp \left(i\left(\omega_{l}+\omega_{l+1}\right)\right) U_{2(l+1) 1 l}^{l} P_{l+1}^{1} \\
& +\sqrt{\frac{l(2 l+1)}{2(l+3)(2 l+3)}} \exp \left(i\left(\omega_{l}+\omega_{l+2}\right)\right) U_{2(l+2) 1 l}^{l} P_{l+2}^{1} \\
& +\sqrt{\frac{l(l-1)}{2(2 l-3)(l-3)}} \exp \left(i\left(\omega_{l}+\omega_{l-3}\right)\right) U_{2(l-3) 1 l}^{l-1} P_{l-3}^{1} \\
& -\frac{1}{2} \exp \left(i\left(\omega_{l}+\omega_{l-2}\right)\right) U_{2(l-2) 1 l}^{l-1} P_{l-2}^{1} \\
& -\sqrt{\frac{3(2 l-1)}{4 l(2 l-3)(2 l+1)}} \exp \left(i\left(\omega_{l}+\omega_{l-1}\right)\right) U_{2(l-1) 1 l}^{l-1} P_{l-1}^{1} \\
& +\sqrt{\frac{l-1}{4 l}} \exp \left(2 i \omega_{l}\right) U_{2 l 1 l}^{l-1} P_{l}^{1} \\
& \left.-\frac{l-1}{\sqrt{2(l+2)(2 l+1)}} \exp \left(i\left(\omega_{l}+\omega_{l+1}\right)\right) U_{2(l+1) 1 l}^{l-1} P_{l+1}^{1}\right],
\end{aligned}
$$




$$
\begin{aligned}
\mathfrak{M}_{21}^{10}= & \frac{i}{2 k} \sum_{l}\left[\frac{l+1}{\sqrt{l(2 l+1)}} \exp \left(i\left(\omega_{l}+\omega_{l-1^{\prime}}\right)\right) U_{2(l-1) 1 l}^{l+1} P_{l-1}^{1}\right. \\
& -\sqrt{\frac{l+2}{2 l} \exp \left(2 i \omega_{l}\right) U_{2 l 1 l}^{l+1} P_{l}^{1}} \\
& +\sqrt{\frac{3(2 l+3)}{2(l+2)(2 l+1)(2 l+5)}} \exp \left(i\left(\omega_{l}+\omega_{l+1}\right)\right) U_{2(l+1) 1 l}^{l+1} P_{l+1}^{1} \\
& +\frac{l+1}{\sqrt{2(l+2)(l+3)}} \exp \left(i\left(\omega_{l}+\omega_{l+2}\right)\right) U_{2(l+2) 1 l}^{l+1} P_{l+2}^{1} \\
& -\sqrt{\frac{(l+2)(l+1)}{(l+3)(2 l+5)}} \exp \left(i\left(\omega_{l}+\omega_{l+3}\right)\right) U_{2(l+3) 1 l}^{l+1} P_{l+3}^{1} \\
& -\sqrt{\frac{l(l-1)}{(2 l-3)(l-2)}} \exp \left(i\left(\omega_{l}+\omega_{l-3}\right)\right) U_{2(l-3) 1 l}^{l-1} P_{l-3}^{1} \\
& +\frac{\sqrt{2(l-1)(l-2)}}{\sqrt{2(2 l-1)}} \exp \left(i\left(\omega_{l}+\omega_{l-2}\right)\right) U_{2(l-2) 1 l}^{l-1} P_{l-2}^{1} \\
& -\sqrt{\frac{l}{2(2 l-3)(2 l+1)(l-1)}} \exp \left(i\left(\omega_{l}+\omega_{l-1}\right)\right) U_{2(l-1) 1 l}^{l-1} P_{l-1}^{1} \\
& -\sqrt{\frac{l-1}{2(l+1)} \exp \left(2 i \omega_{l}\right) U_{2 l 1 l}^{l-1} P_{l}^{1}} \\
& \left.-\frac{l}{\sqrt{(l+1)(2 l+1)}} \exp \left(i\left(\omega_{l}+\omega_{l+1}\right)\right) U_{2(l+1) 1 l}^{l-1} P_{l+1}^{1}\right],
\end{aligned}
$$




$$
\begin{aligned}
& \mathfrak{M}_{2}^{1-1}=-\frac{i}{2 k} \sum_{l}\left[\frac{(l+2) \sqrt{l-2}}{\sqrt{2 l(2 l+1)(l+1)}} \exp \left(i\left(\omega_{l}+\omega_{l-1^{\prime}}\right)\right) U_{2(l-1) 1 l}^{l+1} P_{l-1}^{1}\right. \\
& +\frac{(l+4) \sqrt{l-1}}{4(l+1) \sqrt{l}} \exp \left(2 i \omega_{l}\right) U_{2 l 1 l}^{l+1} P_{l}^{1} \\
& +3 \sqrt{\frac{3 l(l+3)(2 l+3)}{2(l+1)^{2}(l+2)(2 l+1)(2 l+5)}} \exp \left(i\left(\omega_{l}+\omega_{l+1}\right)\right) U_{2(l+1) 1 l}^{l+1} P_{l+1}^{1} \\
& -\frac{(l-1) \sqrt{l+4}}{\sqrt{2(l+1)(l+2)(l+3)}} \exp \left(i\left(\omega_{l}+\omega_{l+2}\right)\right) U_{2(l+2) 1 l}^{l+1} P_{l+2}^{1} \\
& -\sqrt{\frac{(l+1)(l+5)}{2(l+3)(2 l+5)}} \exp \left(i\left(\omega_{l}+\omega_{l+3}\right)\right) U_{2(l+3) 1 l}^{l+1} P_{l+3}^{1} \\
& -\sqrt{\frac{(2 l+1)(l+1)(l-3)}{2 l(l-1)(2 l-1)}} \exp \left(i\left(\omega_{l}+\omega_{l-2}\right)\right) U_{2(l-2) 1 l}^{l} P_{l-2}^{1} \\
& -\frac{(l+3) \sqrt{(2 l+1)(l-2)}}{2 l \sqrt{(l-1)(l+1)}} \exp \left(i\left(\omega_{l}+\omega_{l-1}\right)\right) U_{2(l-1) 1 l}^{l} P_{l-1}^{1} \\
& -\frac{3(2 l+1) \sqrt{3(l+2)(l-1)}}{l(l+1) \sqrt{2(2 l-1)(2 l+3)}} \exp \left(2 i \omega_{l}\right) U_{2 l 1 l}^{l} P_{l}^{1} \\
& -\frac{(l-2) \sqrt{(2 l+1)(l+3)}}{2(l+1) \sqrt{l(l+2)}} \exp \left(i\left(\omega_{l}+\omega_{l+1}\right)\right) U_{2(l+1) 1 l}^{l} P_{l+1}^{1} \\
& +\sqrt{\frac{l(l+4)(2 l+1)}{2(l+1)(l+2)(2 l+3)}} \exp \left(i\left(\omega_{l}+\omega_{l+2}\right)\right) U_{2(l+2) 1 l}^{l} P_{l+2}^{1} \\
& +\sqrt{\frac{l(l-4)}{2(2 l-3)(l-2)}} \exp \left(i\left(\omega_{l}+\omega_{l-3}\right)\right) U_{2(l-3) 1 l}^{l-1} P_{l-3}^{1} \\
& +\frac{(l+2) \sqrt{l-3}}{2 \sqrt{l(l-1)(l-2)}} \exp \left(i\left(\omega_{l}+\omega_{l-2}\right)\right) U_{2(l-2) 1 l}^{l-1} P_{l-2}^{1} \\
& +\frac{3 \sqrt{3(l+1)(l-2)(2 l-1)}}{2 l \sqrt{(l-1)(l-2)}} \exp \left(i\left(\omega_{l}+\omega_{l-1}\right)\right) U_{2(l-1) 1 l}^{l-1} P_{l-1}^{1} \\
& -\frac{(l-3) \sqrt{(l+2)}}{2 l \sqrt{(l+1)}} \exp \left(2 i \omega_{l}\right) U_{2 l 1 l}^{l-1} P_{l}^{1} \\
& \left.-\frac{(l-1) \sqrt{l+3}}{\sqrt{2 l(l+1)(2 l+1)}} \exp \left(i\left(\omega_{l}+\omega_{l+1}\right)\right) U_{2(l+1) 1 l}^{l-1} P_{l+1}^{1}\right] \text {, }
\end{aligned}
$$




$$
\begin{aligned}
& \mathfrak{M}_{20}^{11}=\frac{i}{2 k} \sum_{l}\left[\frac{l+2}{\sqrt{4 l(2 l+1)}} \exp \left(i\left(\omega_{l}+\omega_{l-1^{\prime}}\right)\right) U_{2(l-1) 1 l}^{l+1} P_{l-1}^{1}\right. \\
& +\sqrt{\frac{3(l+2)}{2 l(l+1)^{2}}} \exp \left(2 i \omega_{l}\right) U_{2 l 1 l}^{l+1} P_{l}^{1} \\
& +\frac{\sqrt{(l+2)(2 l+3)}(3-(l+1)(l+2))}{\sqrt{2(2 l+1)(2 l+5)}} \exp \left(i\left(\omega_{l}+\omega_{l+1}\right)\right) U_{2(l+1) 1 l}^{l+1} P_{l+1}^{1} \\
& -\frac{1}{\sqrt{2(l+2)(l+3)}} \exp \left(i\left(\omega_{l}+\omega_{l+2}\right)\right) U_{2(l+2) 1 l}^{l+1} P_{l+2}^{1} \\
& +\sqrt{\frac{3(l+2)(l+1)}{4(l+3)(2 l+5)}} \exp \left(i\left(\omega_{l}+\omega_{l+3}\right)\right) U_{2(l+3) 1 l}^{l+1} P_{l+3}^{1} \\
& -\sqrt{\frac{(2 l+1)(l+1)}{4(2 l-1)(l-1)}} \exp \left(i\left(\omega_{l}+\omega_{l-2}\right)\right) U_{2(l-2) 1 l}^{l} P_{l-2}^{1} \\
& -\sqrt{\frac{3(2 l+1)}{2 l^{2}(l-1)}} \exp \left(i\left(\omega_{l}+\omega_{l-1}\right)\right) U_{2(l-1) 1 l}^{l} P_{l-1}^{1} \\
& -\frac{(2 l+1)(3-l(l+1))}{l(l+1) \sqrt{2(2 l+3)(2 l-1)}} \exp \left(2 i \omega_{l}\right) U_{2 l 1 l}^{l} P_{l}^{1} \\
& -\sqrt{\frac{3(2 l+1)}{2(l+1)^{2}(l+2)}} \exp \left(i\left(\omega_{l}+\omega_{l+1}\right)\right) U_{2(l+1) 1 l}^{l} P_{l+1}^{1} \\
& -\sqrt{\frac{3 l(2 l+1)}{4 l+2(2 l+3)}} \exp \left(i\left(\omega_{l}+\omega_{l+2}\right)\right) U_{2(l+2) 1 l}^{l} P_{l+2}^{1} \\
& +\sqrt{\frac{l(l-1)}{4(l-2)(2 l-3)}} \exp \left(i\left(\omega_{l}+\omega_{l-3}\right)\right) U_{2(l-3) 1 l}^{l-1} P_{l-3}^{1} \\
& +\sqrt{\frac{3}{2(l-1)(l-2)}} \exp \left(i\left(\omega_{l}+\omega_{l-2}\right)\right) U_{2(l-2) 1 l}^{l-1} P_{l-2}^{1} \\
& +\frac{\sqrt{(2 l-1)}(3-l(l-1))}{l \sqrt{2(l-1)(2 l+1)(2 l-3)}} \exp \left(i\left(\omega_{l}+\omega_{l-1}\right)\right) U_{2(l-1) 1 l}^{l-1} P_{l-1}^{1} \\
& +\sqrt{\frac{3(l-1)}{2 l^{2}(l+1)}} \exp \left(2 i \omega_{l}\right) U_{2 l 1 l}^{l-1} P_{l}^{1} \\
& \left.+\frac{\sqrt{3}(l-1)}{2 \sqrt{(2 l+1)(l+1)}} \exp \left(i\left(\omega_{l}+\omega_{l+1}\right)\right) U_{2(l+1) 1 l}^{l-1} P_{l+1}^{1}\right],
\end{aligned}
$$




$$
\begin{aligned}
& \mathfrak{M}_{11}^{22}=-\frac{i}{2 k} \sum_{l}\left[\frac{(l+3)(l+4)}{2(l+2) \sqrt{2(2 l+3)(l+1)}} \exp \left(i\left(\omega_{l}+\omega_{l+1}\right)\right) U_{1(l+1) 2 l}^{l+2} P_{l+1}^{1}\right. \\
& -\frac{(l+4) \sqrt{(l+1)(2 l+5)}}{2(l+2) \sqrt{2(2 l+3)(l+1)}} \exp \left(i\left(\omega_{l}+\omega_{l+2}\right)\right) U_{1(l+2) 2 l}^{l+2} P_{l+2}^{1} \\
& +\sqrt{\frac{(l+1)(l+2)}{8(2 l+3)(l+3)}} \exp \left(i\left(\omega_{l}+\omega_{l+3}\right)\right) U_{1(l+3) 2 l}^{l+2} P_{l+3}^{1} \\
& -\frac{(l+3) \sqrt{l+2}}{(l+1) \sqrt{2 l}} \exp \left(2 i \omega_{l}\right) U_{1 l 2 l}^{l+1} P_{l}^{1} \\
& +\frac{(l+3) \sqrt{l(2 l+3)}}{4(l+1)(l+2)} \exp \left(i\left(\omega_{l}+\omega_{l+1}\right)\right) U_{1(l+1) 2 l}^{l+1} P_{l+1}^{1} \\
& -\frac{\sqrt{l(l+1)}}{2(l+2)} \exp \left(i\left(\omega_{l}+\omega_{l+2}\right)\right) U_{1(l+2) 2 l}^{l+1} P_{l+2}^{1} \\
& +\sqrt{\frac{3(l+1)(l+2)(2 l+1)}{4 l^{2}(2 l+3)(2 l-1)}} \exp \left(i\left(\omega_{l}+\omega_{l-1}\right)\right) U_{1(l-1) 2 l}^{l} P_{l-1}^{1} \\
& -\frac{3(l+2)(l-1)(2 l+1)}{l(l+1) \sqrt{(2 l+3)(2 l-1)}} \exp \left(2 i \omega_{l}\right) U_{1 l 2 l}^{l} P_{l}^{1} \\
& +\frac{(l-1) \sqrt{3 l(2 l+1)}}{2(l+1) \sqrt{(2 l+3)(2 l-1)}} \exp \left(i\left(\omega_{l}+\omega_{l+1}\right)\right) U_{1(l+1) 2 l}^{l} P_{l+1}^{1} \\
& -\frac{\sqrt{l(l+1)}}{2(l-1)} \exp \left(i\left(\omega_{l}+\omega_{l-2}\right)\right) U_{1(l-2) 2 l}^{l-1} P_{l-2}^{1} \\
& +\frac{\sqrt{(l+1)(l-2)(2 l-1)}}{2(l-1) \sqrt{l}} \exp \left(i\left(\omega_{l}+\omega_{l-1}\right)\right) U_{1(l-1) 2 l}^{l-1} P_{l-1}^{1} \\
& -\frac{(l-2) \sqrt{l-1}}{2 l \sqrt{l+1}} \exp \left(2 i \omega_{l}\right) U_{1 l 2 l}^{l-1} P_{l}^{1} \\
& +\sqrt{\frac{l(l-1)}{8(l-2)(2 l-1)}} \exp \left(i\left(\omega_{l}+\omega_{l-3}\right)\right) U_{1(l-3) 2 l}^{l-2} P_{l-3}^{1} \\
& -\frac{(l-3) \sqrt{l(2 l-3)}}{2(l-1) \sqrt{(l-2)(2 l-1)}} \exp \left(i\left(\omega_{l}+\omega_{l-2}\right)\right) U_{1(l-2) 2 l}^{l-2} P_{l-2}^{1} \\
& \left.+\frac{(l-3)(l-2)}{2(l-1) \sqrt{2 l(2 l-1)}} \exp \left(i\left(\omega_{l}+\omega_{l-1}\right)\right) U_{1(l-1) 2 l}^{l-2} P_{l-1}^{1}\right]
\end{aligned}
$$




$$
\begin{aligned}
& \mathfrak{M}_{11}^{21}=-\frac{i}{2 k} \sum_{l}\left[\frac{(l+3) \sqrt{(l+1)}}{\sqrt{2(2 l+3)}} \exp \left(i\left(\omega_{l}+\omega_{l+1}\right)\right) U_{1(l+1) 2 l}^{l+2} P_{l+1}^{1}\right. \\
& -\sqrt{\frac{(l+1)(l+3)(2 l+5)}{2(2 l+3)}} \exp \left(i\left(\omega_{l}+\omega_{l+2}\right)\right) U_{1(l+2) 2 l}^{l+2} P_{l+2}^{1} \\
& +\sqrt{\frac{(l+1)(l+2)(l+3)}{2(2 l+3)}} \exp \left(i\left(\omega_{l}+\omega_{l+3}\right)\right) U_{1(l+3) 2 l}^{l+2} P_{l+3}^{1} \\
& -\frac{\sqrt{l(l+2)}}{2} \exp \left(2 i \omega_{l}\right) U_{1 l 2 l}^{l+1} P_{l}^{1} \\
& +\frac{\sqrt{l(2 l+3)}}{2} \exp \left(i\left(\omega_{l}+\omega_{l+1}\right)\right) U_{1(l+1) 2 l}^{l+1} P_{l+1}^{1} \\
& -\frac{\sqrt{l(l+1)}}{2} \exp \left(i\left(\omega_{l}+\omega_{l+2}\right)\right) U_{1(l+2) 2 l}^{l+1} P_{l+2}^{1} \\
& -\sqrt{\frac{3(l+1)(2 l+1)}{4(2 l+3)(2 l-1)}} \exp \left(i\left(\omega_{l}+\omega_{l-1}\right)\right) U_{1(l-1) 2 l}^{l} P_{l-1}^{1} \\
& +\frac{\sqrt{3}(2 l+1)}{2 \sqrt{(2 l+3)(2 l-1)}} \exp \left(2 i \omega_{l}\right) U_{1 l 2 l}^{l} P_{l}^{1} \\
& -\sqrt{\frac{3 l(2 l+1)}{4(2 l+3)(2 l-1)}} \exp \left(i\left(\omega_{l}+\omega_{l+1}\right)\right) U_{1(l+1) 2 l}^{l} P_{l+1}^{1} \\
& +\frac{\sqrt{l(l+1)}}{2} \exp \left(i\left(\omega_{l}+\omega_{l-2}\right)\right) U_{1(l-2) 2 l}^{l-1} P_{l-2}^{1} \\
& -\frac{\sqrt{(l+1)(2 l-1)}}{2} \exp \left(i\left(\omega_{l}+\omega_{l-1}\right)\right) U_{1(l-1) 2 l}^{l-1} P_{l-1}^{1} \\
& +\frac{\sqrt{(l-1)(l+1)}}{2} \exp \left(2 i \omega_{l}\right) U_{1 l 2 l}^{l-1} P_{l}^{1} \\
& -\sqrt{\frac{l(l-2)(l-1)}{2(2 l-1)}} \exp \left(i\left(\omega_{l}+\omega_{l-3}\right)\right) U_{1(l-3) 2 l}^{l-2} P_{l-3}^{1} \\
& +\frac{\sqrt{l(l-2)(2 l-3)}}{\sqrt{2(2 l-1)}} \exp \left(i\left(\omega_{l}+\omega_{l-2}\right)\right) U_{1(l-2) 2 l}^{l-2} P_{l-2}^{1} \\
& \left.-\frac{(l-2) \sqrt{l}}{\sqrt{2(2 l-1)}} \exp \left(i\left(\omega_{l}+\omega_{l-1}\right)\right) U_{1(l-1) 2 l}^{l-2} P_{l-1}^{1}\right] \text {, }
\end{aligned}
$$




$$
\begin{aligned}
& \mathfrak{M}_{11}^{20}=\frac{i}{2 k} \sum_{l}\left[\sqrt{\frac{3(l+1)}{4(2 l+3)}} \exp \left(i\left(\omega_{l}+\omega_{l+1}\right)\right) U_{1(l+1) 2 l}^{l+2} P_{l+1}^{1}\right. \\
& -\sqrt{\frac{3(l+1)(2 l+5)}{4(2 l+3)(l+3)}} \exp \left(i\left(\omega_{l}+\omega_{l+2}\right)\right) U_{1(l+2) 2 l}^{l+2} P_{l+2}^{1} \\
& +\sqrt{\frac{3(l+1)(l+2)}{4(l+3)(2 l+3)}} \exp \left(i\left(\omega_{l}+\omega_{l+3}\right)\right) U_{1(l+3) 2 l}^{l+2} P_{l+3}^{1} \\
& -\sqrt{\frac{(2 l+1)(l+1)}{2(2 l+3)(2 l-1)}} \exp \left(i\left(\omega_{l}+\omega_{l-1}\right)\right) U_{1(l-1) 2 l}^{l} P_{l-1}^{1} \\
& +\frac{2 l+1}{\sqrt{(2 l+3)(2 l-1)}} \exp \left(2 i \omega_{l}\right) U_{1 l 2 l}^{l} P_{l}^{1} \\
& -\sqrt{\frac{l(2 l+1)}{(2 l+3)(2 l-1)}} \exp \left(i\left(\omega_{l}+\omega_{l+1}\right)\right) U_{1(l+1) 2 l}^{l} P_{l+1}^{1} \\
& +\sqrt{\frac{3 l(l-1)}{4(l-2)(2 l-1)}} \exp \left(i\left(\omega_{l}+\omega_{l-3}\right)\right) U_{1(l-3) 2 l}^{l-2} P_{l-3}^{1} \\
& -\sqrt{\frac{3 l(2 l-3)}{2(l-2)(2 l-1)}} \exp \left(i\left(\omega_{l}+\omega_{l-2}\right)\right) U_{1(l-2) 2 l}^{l-2} P_{l-2}^{1} \\
& \left.+\sqrt{\frac{3 l}{2(2 l-1)}} \exp \left(i\left(\omega_{l}+\omega_{l-1}\right)\right) U_{1(l-1) 2 l}^{l-2} P_{l-1}^{1}\right] \text {, }
\end{aligned}
$$




$$
\begin{aligned}
& \mathfrak{M}_{1}^{2-1}=\frac{i}{2 k} \sum_{l}\left[\frac{\sqrt{l+1}}{(l+2) \sqrt{2(2 l+3)}} \exp \left(i\left(\omega_{l}+\omega_{l+1}\right)\right) U_{1(l+1) 2 l}^{l+2} P_{l+1}^{2}\right. \\
& +\frac{\sqrt{(l+1)(2 l+5)}}{(l+2) \sqrt{2(2 l+3)(l+3)}} \exp \left(i\left(\omega_{l}+\omega_{l+2}\right)\right) U_{1(l+2) 2 l}^{l+2} P_{l+2}^{2} \\
& +\sqrt{\frac{l+1}{2(l+3)(l+2)(2 l+3)}} \exp \left(i\left(\omega_{l}+\omega_{l+3}\right)\right) U_{1(l+3) 2 l}^{l+2} P_{l+3}^{2} \\
& -\frac{\sqrt{l}}{2(l+1) \sqrt{(l+2)}} \exp \left(2 i \omega_{l}\right) U_{1 l 2 l}^{l+1} P_{l}^{2} \\
& -\frac{\sqrt{l(2 l+3)}}{2(l+1)(l+2)} \exp \left(i\left(\omega_{l}+\omega_{l+1}\right)\right) U_{1(l+1) 2 l}^{l+1} P_{l+1}^{2} \\
& -\frac{\sqrt{l}}{2(l+2 \sqrt{l+1})} \exp \left(i\left(\omega_{l}+\omega_{l+2}\right)\right) U_{1(l+2) 2 l}^{l+1} P_{l+2}^{2} \\
& -\frac{\sqrt{3(2 l+1)}}{2 l \sqrt{(2 l+3)(2 l-1)(l+1)}} \exp \left(i\left(\omega_{l}+\omega_{l-1}\right)\right) U_{1(l-1) 2 l}^{l} P_{l-1}^{2} \\
& -\frac{\sqrt{3(2 l+1)}}{2 l \sqrt{(2 l+3)(2 l-1)(l+1)}} \exp \left(2 i \omega_{l}\right) U_{1 l 2 l}^{l} P_{l}^{2} \\
& -\frac{\sqrt{3(2 l+1)}}{2(l+1) \sqrt{l(2 l+3)(2 l-1)}} \exp \left(i\left(\omega_{l}+\omega_{l+1}\right)\right) U_{1(l+1) 2 l}^{l} P_{l+1}^{2} \\
& +\frac{\sqrt{l+1}}{2(l-1) \sqrt{l}} \exp \left(i\left(\omega_{l}+\omega_{l-2}\right)\right) U_{1(l-2) 2 l}^{l-1} P_{l-2}^{2} \\
& +\frac{\sqrt{(l+1)(2 l-1)}}{2 l(l-1)} \exp \left(i\left(\omega_{l}+\omega_{l-1}\right)\right) U_{1(l-1) 2 l}^{l-1} P_{l-1}^{2} \\
& +\frac{\sqrt{l+1}}{2 l \sqrt{l-1}} \exp \left(2 i \omega_{l}\right) U_{1 l 2 l}^{l-1} P_{l}^{2} \\
& -\sqrt{\frac{l}{2(2 l-1)(l-1)(l-2)}} \exp \left(i\left(\omega_{l}+\omega_{l-3}\right)\right) U_{1(l-3) 2 l}^{l-2} P_{l-3}^{2} \\
& -\frac{\sqrt{l(2 l-3)}}{(l-1) \sqrt{2(2 l-1)(l-2)}} \exp \left(i\left(\omega_{l}+\omega_{l-2}\right)\right) U_{1(l-2) 2 l}^{l-2} P_{l-2}^{2} \\
& \left.-\frac{\sqrt{l}}{(l-1) \sqrt{2(2 l-1)}} \exp \left(i\left(\omega_{l}+\omega_{l-1}\right)\right) U_{1(l-1) 2 l}^{l-2} P_{l-1}^{2}\right] \text {, }
\end{aligned}
$$




$$
\begin{aligned}
& \mathfrak{M}_{1}^{2-2}=\frac{i}{2 k} \sum_{l}\left[\frac{1}{2(l+2) \sqrt{2(2 l+3)(l+1)}} \exp \left(i\left(\omega_{l}+\omega_{l+1}\right)\right) U_{1(l+1) 2 l}^{l+2} P_{l+1}^{3}\right. \\
& +\frac{1}{2(l+2) \sqrt{(2 l+3)(l+1)(l+3)}} \exp \left(i\left(\omega_{l}+\omega_{l+2}\right)\right) U_{1(l+2) 2 l}^{l+2} P_{l+2}^{3} \\
& +\frac{1}{\sqrt{8(l+3)(l+2)(l+1)(2 l+3)}} \exp \left(i\left(\omega_{l}+\omega_{l+3}\right)\right) U_{1(l+3) 2 l}^{l+2} P_{l+3}^{3} \\
& -\frac{1}{2(l+1) \sqrt{l(l+2)}} \exp \left(2 i \omega_{l}\right) U_{1 l 2 l}^{l+1} P_{l}^{3} \\
& -\frac{\sqrt{(2 l+3)}}{2(l+1)(l+2) \sqrt{l}} \exp \left(i\left(\omega_{l}+\omega_{l+1}\right)\right) U_{1(l+1) 2 l}^{l+1} P_{l+1}^{3} \\
& -\frac{1}{2(l+2) \sqrt{l(l+1)}} \exp \left(i\left(\omega_{l}+\omega_{l+2}\right)\right) U_{1(l+2) 2 l}^{l+1} P_{l+2}^{3} \\
& +\frac{\sqrt{3(2 l+1)}}{2 l \sqrt{(2 l+3)(2 l-1)(l+1)}} \exp \left(i\left(\omega_{l}+\omega_{l-1}\right)\right) U_{1(l-1) 2 l}^{l} P_{l-1}^{3} \\
& +\frac{(2 l+1) \sqrt{3}}{2 l(l+1) \sqrt{(2 l+3)(2 l-1)}} \exp \left(2 i \omega_{l}\right) U_{1 l 2 l}^{l} P_{l}^{3} \\
& +\frac{\sqrt{3(2 l+1)}}{2(l+1) \sqrt{l(2 l+3)(2 l-1)}} \exp \left(i\left(\omega_{l}+\omega_{l+1}\right)\right) U_{1(l+1) 2 l}^{l} P_{l+1}^{3} \\
& -\frac{1}{2(l-1) \sqrt{l(l+1)}} \exp \left(i\left(\omega_{l}+\omega_{l-2}\right)\right) U_{1(l-2) 2 l}^{l-1} P_{l-2}^{3} \\
& -\frac{\sqrt{(2 l-1)}}{2(l-1) l(l+1)} \exp \left(i\left(\omega_{l}+\omega_{l-1}\right)\right) U_{1(l-1) 2 l}^{l-1} P_{l-1}^{3} \\
& +\frac{1}{2 l \sqrt{(l-1)(l+1)}} \exp \left(2 i \omega_{l}\right) U_{1 l 2 l}^{l-1} P_{l}^{3} \\
& +\frac{1}{\sqrt{8(2 l-1) l(l-1)(l-2)}} \exp \left(i\left(\omega_{l}+\omega_{l-3}\right)\right) U_{1(l-3) 2 l}^{l-2} P_{l-3}^{3} \\
& +\frac{\sqrt{(2 l-3)}}{2(l-1) \sqrt{l(2 l-1)(l-2)}} \exp \left(i\left(\omega_{l}+\omega_{l-2}\right)\right) U_{1(l-2) 2 l}^{l-2} P_{l-2}^{3} \\
& \left.+\frac{1}{2(l-1) \sqrt{2 l(2 l-1)}} \exp \left(i\left(\omega_{l}+\omega_{l-1}\right)\right) U_{1(l-1) 2 l}^{l-2} P_{l-1}^{3}\right] \text {, }
\end{aligned}
$$




$$
\begin{aligned}
& \mathfrak{M}_{10}^{22}=-\frac{i}{2 k} \sum_{l}\left[\frac{l+4}{2(l+2) \sqrt{(l+1)(2 l+3)}} \exp \left(i\left(\omega_{l}+\omega_{l+1}\right)\right) U_{1(l+1) 2 l}^{l+2} P_{l+1}^{2}\right. \\
& +\frac{\sqrt{(2 l+5)}}{(l+2) \sqrt{(2 l+3)(l+3)(l+1)}} \exp \left(i\left(\omega_{l}+\omega_{l+2}\right)\right) U_{1(l+2) 2 l}^{l+2} P_{l+2}^{2} \\
& +\sqrt{\frac{(l+1)(l+3)}{4(l+2)(2 l+3)}} \exp \left(i\left(\omega_{l}+\omega_{l+3}\right)\right) U_{1(l+3) 2 l}^{l+2} P_{l+3}^{2} \\
& -\frac{l+3}{\sqrt{2} l(l+2)(l-1)} \exp \left(2 i \omega_{l}\right) U_{1 l 2 l}^{l+1} P_{l}^{2} \\
& -\frac{\sqrt{2(2 l+3)}}{(l+1)(l+2) \sqrt{l}} \exp \left(i\left(\omega_{l}+\omega_{l+1}\right)\right) U_{1(l+1) 2 l}^{l+1} P_{l+1}^{2} \\
& -\frac{\sqrt{l}}{(l+2) \sqrt{2(l+1)}} \exp \left(i\left(\omega_{l}+\omega_{l+2}\right)\right) U_{1(l+2) 2 l}^{l+1} P_{l+2}^{2} \\
& -\frac{(l+2) \sqrt{3(2 l+1)}}{l \sqrt{2(2 l+3)(2 l-1)(l-1)}} \exp \left(i\left(\omega_{l}+\omega_{l-1}\right)\right) U_{1(l-1) 2 l}^{l} P_{l-1}^{2} \\
& +\frac{\sqrt{6}(2 l+1)}{l(l+1) \sqrt{(2 l+3)(2 l-1)}} \exp \left(2 i \omega_{l}\right) U_{1 l 2 l}^{l} P_{l}^{2} \\
& -\frac{(l-1) \sqrt{3(2 l+1)}}{(l+1) \sqrt{2 l(2 l+3)(2 l-1)}} \exp \left(i\left(\omega_{l}+\omega_{l+1}\right)\right) U_{1(l+1) 2 l}^{l} P_{l+1}^{2} \\
& -\frac{\sqrt{l+1}}{(l-1) \sqrt{2 l}} \exp \left(i\left(\omega_{l}+\omega_{l-2}\right)\right) U_{1(l-2) 2 l}^{l-1} P_{l-2}^{2} \\
& -\frac{\sqrt{2(2 l-1)}}{l(l-1) \sqrt{l+1}} \exp \left(i\left(\omega_{l}+\omega_{l-1}\right)\right) U_{1(l-1) 2 l}^{l-1} P_{l-1}^{2} \\
& +\frac{l-2}{l \sqrt{2(l+1)(l-1)}} \exp \left(2 i \omega_{l}\right) U_{1 l 2 l}^{l-1} P_{l}^{2} \\
& +\sqrt{\frac{l}{4(2 l-1)(l-1)(l-2)(l-4)}} \exp \left(i\left(\omega_{l}+\omega_{l-3}\right)\right) U_{1(l-3) 2 l}^{l-2} P_{l-3}^{2} \\
& +\frac{\sqrt{2 l-3}}{(l-1) \sqrt{l(2 l-1)(l-2)}} \exp \left(i\left(\omega_{l}+\omega_{l-2}\right)\right) U_{1(l-2) 2 l}^{l-2} P_{l-2}^{2} \\
& \left.-\frac{l-3}{2 l(l-1) \sqrt{2 l-1}} \exp \left(i\left(\omega_{l}+\omega_{l-1}\right)\right) U_{1(l-1) 2 l}^{l-2} P_{l-1}^{2}\right] \text {, }
\end{aligned}
$$




$$
\begin{aligned}
& \mathfrak{M}_{10}^{21}=-\frac{i}{2 k} \sum_{l}\left[\frac{(l+3) \sqrt{l+1}}{(l+2) \sqrt{2 l+3}} \exp \left(i\left(\omega_{l}+\omega_{l+1}\right)\right) U_{1(l+1) 2 l}^{l+2} P_{l+1}^{1}\right. \\
& +\frac{\sqrt{(l+1)(2 l+5)}}{(l+2) \sqrt{(l+3)(2 l+3)}} \exp \left(i\left(\omega_{l}+\omega_{l+2}\right)\right) U_{1(l+2) 2 l}^{l+2} P_{l+2}^{1} \\
& -\sqrt{\frac{(l+1)(l+2)}{(l+3)(2 l+3)}} \exp \left(i\left(\omega_{l}+\omega_{l+3}\right)\right) U_{1(l+3) 2 l}^{l+2} P_{l+3}^{1} \\
& -\frac{\sqrt{l(l+2)}}{\sqrt{2}(l+1)} \exp \left(2 i \omega_{l}\right) U_{1 l 2 l}^{l+1} P_{l}^{1} \\
& -\frac{\sqrt{l(2 l+3)}}{\sqrt{2}(l+1)(l+2)} \exp \left(i\left(\omega_{l}+\omega_{l+1}\right)\right) U_{1(l+1) 2 l}^{l+1} P_{l+1}^{1} \\
& -\frac{\sqrt{l(l+1)}}{\sqrt{2}(l+2)} \exp \left(i\left(\omega_{l}+\omega_{l+2}\right)\right) U_{1(l+2) 2 l}^{l+1} P_{l+2}^{1} \\
& -\sqrt{\frac{3(l+1)(2 l+1)}{2 l^{2}(2 l+3)(2 l-1)}} \exp \left(i\left(\omega_{l}+\omega_{l-1}\right)\right) U_{1(l-1) 2 l}^{l} P_{l-1}^{1} \\
& -\frac{\sqrt{3}(2 l+1)}{l(l+1) \sqrt{2(2 l+3)(2 l-1)}} \exp \left(2 i \omega_{l}\right) U_{1 l 2 l}^{l} P_{l}^{1} \\
& +\frac{\sqrt{3 l(2 l+1)}}{(l+1) \sqrt{2(2 l+3)(2 l-1)}} \exp \left(i\left(\omega_{l}+\omega_{l+1}\right)\right) U_{1(l+1) 2 l}^{l} P_{l+1}^{1} \\
& +\frac{\sqrt{l(l+1)}}{\sqrt{2}(l-1)} \exp \left(i\left(\omega_{l}+\omega_{l-2}\right)\right) U_{1(l-2) 2 l}^{l-1} P_{l-2}^{1} \\
& +\frac{\sqrt{(l+1)(2 l-1)}}{\sqrt{2} l(l-1)} \exp \left(i\left(\omega_{l}+\omega_{l-1}\right)\right) U_{1(l-1) 2 l}^{l-1} P_{l-1}^{1} \\
& -\frac{\sqrt{(l-1)(l+1)}}{\sqrt{2} l} \exp \left(2 i \omega_{l}\right) U_{1 l 2 l}^{l-1} P_{l}^{1} \\
& -\sqrt{\frac{l(l-1)}{(2 l-1)(l-2)}} \exp \left(i\left(\omega_{l}+\omega_{l-3}\right)\right) U_{1(l-3) 2 l}^{l-2} P_{l-3}^{1} \\
& -\frac{\sqrt{l(2 l-3)}}{(l-1) \sqrt{(2 l-1)(l-2)}} \exp \left(i\left(\omega_{l}+\omega_{l-2}\right)\right) U_{1(l-2) 2 l}^{l-2} P_{l-2}^{1} \\
& \left.+\frac{(l-2) \sqrt{l}}{(l-1) \sqrt{2(2 l-1)}} \exp \left(i\left(\omega_{l}+\omega_{l-1}\right)\right) U_{1(l-1) 2 l}^{l-2} P_{l-1}^{1}\right] \text {. }
\end{aligned}
$$

\section{Appendix B: Partial amplitudes for spin-1-spin- $1 / 2$ system}

Below are defined the partial amplitudes for the doublet and quartet spin states.

\section{Doublet spin state}

The independent partial amplitudes for the doublet spin state are the following:

$$
\mathcal{D}_{1 / 2}^{1 / 2} \underset{1 / 2}{1 / 2}=f_{c}(\theta)+\frac{1}{2 i k} \sum_{l=0}\left[(l+1) \alpha^{l+1 / 2}+l \alpha^{l-1 / 2}\right] \exp \left(2 i \omega_{l}\right) P_{l}(\cos \theta),
$$




$$
\mathcal{D}_{1 / 2}^{1 / 2-1 / 2}=-\frac{1}{2 i k} \sum_{l=1} \sqrt{l(l+1)}\left[\alpha^{l+1 / 2}-\alpha^{l-1 / 2}\right] \exp \left(2 i \omega_{l}\right) P_{l}^{1}(\cos \theta)
$$

\section{Quartet spin states}

The independent partial amplitudes for the quartet spin state are the following:

$$
\begin{aligned}
& \mathcal{Q}_{1 / 2}^{3 / 2} \underset{1 / 2}{1 / 2}=f_{c}(\theta)+\frac{1}{4 i k} \sum_{l=0}\left[\frac{3(l+1)(l+2)}{2 l+3} \alpha^{l+3 / 2}+\frac{l(l+1)}{2 l+3} \alpha^{l+1 / 2}+\frac{l(l+1)}{2 l-1} \alpha^{l-1 / 2}\right. \\
& \left.+\frac{3 l(l-1)}{2 l-1} \alpha^{l-3 / 2}\right] \exp \left(2 i \omega_{l}\right) P_{l}(\cos \theta) \\
& \mathcal{Q}_{3 / 2}^{3 / 2}=f_{c}(\theta)+\frac{1}{4 i k} \sum_{l=0}\left[\frac{(l+2)(l+3)}{2 l+3} \alpha^{l+3 / 2}+\frac{3(l+1)(l+2)}{2 l+3} \alpha^{l+1 / 2}+\frac{3 l(l-1)}{2 l-1} \alpha^{l-1 / 2}\right. \\
& \left.+\frac{(l-1)(l-2)}{2 l-1} \alpha^{l-3 / 2}\right] \exp \left(2 i \omega_{l}\right) P_{l}(\cos \theta) \\
& \mathcal{Q}_{1 / 2}^{3 / 2-1 / 2}=-\frac{1}{4 i k} \sum_{l=1}\left[\frac{3(l+2)}{2 l+3} \alpha^{l+3 / 2}-\frac{l+3}{2 l+3} \alpha^{l+1 / 2}+\frac{l-2}{2 l-1} \alpha^{l-1 / 2}-\frac{3(l-1)}{2 l-1} \alpha^{l-3 / 2}\right] \\
& \times \exp \left(2 i \omega_{l}\right) P_{l}^{1}(\cos \theta), \\
& \mathcal{Q}_{3 / 2}^{3 / 2} \underset{3 / 2}{1 / 2}=-\frac{\sqrt{3}}{4 i k} \sum_{l=1}\left[\frac{l+2}{2 l+3} \alpha^{l+3 / 2}+\frac{l+1}{2 l+3} \alpha^{l+1 / 2}-\frac{l}{2 l-1} \alpha^{l-1 / 2}-\frac{l-1}{2 l-1} \alpha^{l-3 / 2}\right] \\
& \times \exp \left(2 i \omega_{l}\right) P_{l}^{1}(\cos \theta), \\
& \mathcal{Q}_{3 / 2}^{3 / 2-1 / 2}=-\frac{\sqrt{3}}{4 i k} \sum_{l=1}\left[\frac{(l+2)(l+3)}{(2 l+3)(l+1)} \alpha^{l+3 / 2}+\frac{(l+2)(l-3)}{l(2 l+3)} \alpha^{l+1 / 2}-\frac{(l+4)(l-1)}{(2 l-1)(l+1)} \alpha^{l-1 / 2}\right. \\
& \left.-\frac{(l-1)(l-2)}{l(2 l-1)} \alpha^{l-3 / 2}\right] \exp \left(2 i \omega_{l}\right) P_{l}(\cos \theta) \text {, } \\
& \mathcal{Q}_{1 / 2}^{3 / 2}=\frac{\sqrt{3}}{4 i k} \sum_{l=2}^{3 / 2}\left[\frac{1}{2 l+3} \alpha^{l+3 / 2}-\frac{1}{2 l+3} \alpha^{l+1 / 2}-\frac{1}{2 l-1} \alpha^{l-1 / 2}+\frac{1}{2 l-1} \alpha^{l-3 / 2}\right] \\
& \times \exp \left(2 i \omega_{l}\right) P_{l}^{2}(\cos \theta), \\
& \mathcal{Q}_{-1 / 2}^{3 / 23 / 2}=\frac{\sqrt{3}}{4 i k} \sum_{l=2}\left[\frac{l+3}{(2 l+3)(l+1)} \alpha^{l+3 / 2}-\frac{l+6}{l(2 l+3)} \alpha^{l+1 / 2}-\frac{l-5}{(2 l-1)(l+1)} \alpha^{l-1 / 2}\right. \\
& \left.+\frac{l-2}{l(2 l-1)} \alpha^{l-3 / 2}\right] \exp \left(2 i \omega_{l}\right) P_{l}^{2}(\cos \theta) \text {, } \\
& \mathcal{Q}_{-3 / 2}^{3 / 23 / 2}=-\frac{1}{4 i k} \sum_{l=3}\left[\frac{1}{(2 l+3)(l+1)} \alpha^{l+3 / 2}-\frac{3}{l(2 l+3)} \alpha^{l+1 / 2}+\frac{3}{(2 l-1)(l+1)} \alpha^{l-1 / 2}\right. \\
& \left.-\frac{1}{l(2 l-1)} \alpha^{l-3 / 2}\right]\left(2 i \omega_{l}\right) P_{l}^{3}(\cos \theta) \text {. }
\end{aligned}
$$


3. Spin-mixing $M$-matrix elements for half-integer channel spin $S=1 / 2$ and $S=3 / 2$

$$
\begin{aligned}
& \mathfrak{M}_{3 / 2}^{1 / 2} \frac{1 / 2}{3 / 2}=\frac{i}{2 k} \sum_{l}\left[\frac{l+1}{\sqrt{2 l(2 l+1)}} \exp \left(i\left(\omega_{l}+\omega_{l-1}\right)\right) U_{3 / 2(l-1) 1 / 2 l}^{l+1 / 2} P_{l-1}^{1}\right. \\
& -\frac{\sqrt{3}(l+1)}{\sqrt{2 l(2 l+3)}} \exp \left(2 i \omega_{l}\right) U_{3 / 2 l 1 / 2 l}^{l+1 / 2} P_{l}^{1} \\
& -\frac{\sqrt{3}(l+1)}{\sqrt{2(l+2)(2 l+3)}} \exp \left(i\left(\omega_{l}+\omega_{l+1}\right)\right) U_{3 / 2(l+1) 1 / 2 l}^{l+1 / 2} P_{l+1}^{1} \\
& -\frac{l+1}{\sqrt{2(l+2)(2 l+3)}} \exp \left(i\left(\omega_{l}+\omega_{l+2}\right)\right) U_{3 / 2(l+2) 1 / 2 l}^{l+1 / 2} P_{l+2}^{1} \\
& -\frac{l}{\sqrt{2(l-1)(2 l-1)}} \exp \left(i\left(\omega_{l}+\omega_{l-2}\right)\right) U_{3 / 2(l-2) 1 / 2 l}^{l-1 / 2} P_{l-2}^{1} \\
& +\frac{\sqrt{3} l}{\sqrt{2(l-1)(2 l+1)}} \exp \left(i\left(\omega_{l}+\omega_{l-1}\right)\right) U_{3 / 2(l-1) 1 / 2 l}^{l-1 / 2} P_{l-1}^{1} \\
& -\frac{\sqrt{3} l}{\sqrt{2(l+1)(2 l-1)}} \exp \left(2 i \omega_{l}\right) U_{3 / 2 l 1 / 2 l}^{l-1 / 2} P_{l}^{1} \\
& \left.+\frac{l}{\sqrt{2(l+1)(2 l+1)}} \exp \left(i\left(\omega_{l}+\omega_{l+1}\right)\right) U_{3 / 2(l+1) 1 / 2 l}^{l-1 / 2} P_{l+1}^{1}\right] \\
& \mathfrak{M}_{3 / 23 / 2}^{1 / 2-1 / 2}=\frac{i}{2 k} \sum_{l}\left[\frac{1}{\sqrt{2 l(2 l+1)}} \exp \left(i\left(\omega_{l}+\omega_{l-1}\right)\right) U_{3 / 2(l-1) 1 / 2 l}^{l+1 / 2} P_{l-1}^{2}\right. \\
& +\sqrt{\frac{3(l+1)}{2 l(2 l+3)(l-1)}} \exp \left(2 i \omega_{l}\right) U_{3 / 2 l 1 / 2 l}^{l+1 / 2} P_{l}^{2} \\
& +\sqrt{\frac{3}{(2 l+1)(l+2)}} \exp \left(i\left(\omega_{l}+\omega_{l+1}\right)\right) U_{3 / 2(l+1) 1 / 2 l}^{l+1 / 2} P_{l+1}^{2} \\
& +\frac{1}{\sqrt{2(l+2)(2 l-1)}} \exp \left(i\left(\omega_{l}+\omega_{l+2}\right)\right) U_{3 / 2(l+2) 1 / 2 l}^{l+1 / 2} P_{l+2}^{2} \\
& -\frac{1}{\sqrt{2(l-1)(2 l-1)}} \exp \left(i\left(\omega_{l}+\omega_{l-2}\right)\right) U_{3 / 2(l-2) 1 / 2 l}^{l-1 / 2} P_{l-2}^{2} \\
& -\sqrt{\frac{3 l}{2(2 l+3)(l-1)(l-2)}} \exp \left(i\left(\omega_{l}+\omega_{l-1}\right)\right) U_{3 / 2(l-1) 1 / 2 l}^{l-1 / 2} P_{l-1}^{2} \\
& -\sqrt{\frac{3}{2(2 l-1)(l+1)}} \exp \left(2 i \omega_{l}\right) U_{3 / 2 l 1 / 2 l}^{l-1 / 2} P_{l}^{2} \\
& \left.-\frac{1}{\sqrt{2(l+1)(2 l+1)}} \exp \left(i\left(\omega_{l}+\omega_{l+1}\right)\right) U_{3 / 2(l+1) 1 / 2 l}^{l-1 / 2} P_{l+1}^{2}\right]
\end{aligned}
$$




$$
\begin{aligned}
& \mathfrak{M}_{3 / 21 / 2}^{1 / 21 / 2}=-\frac{i}{2 k} \sum_{l}\left[\frac{\sqrt{3 l}(l+1)}{\sqrt{2(2 l+1)}} \exp \left(i\left(\omega_{l}+\omega_{l-1}\right)\right) U_{3 / 2(l-1) 1 / 2 l}^{l+1 / 2} P_{l-1}^{0}\right. \\
& -\frac{(l+1) \sqrt{l}}{\sqrt{2(2 l+3)}} \exp \left(2 i \omega_{l}\right) U_{3 / 2 l 1 / 2 l}^{l-1 / 2} P_{l}^{0} \\
& -\frac{(l+1) \sqrt{l+2}}{\sqrt{2(2 l+1)}} \exp \left(i\left(\omega_{l}+\omega_{l+1}\right)\right) U_{3 / 2(l+1) 1 / 2 l}^{l+1 / 2} P_{l+1}^{0} \\
& +\frac{(l+1) \sqrt{3(l+2)}}{\sqrt{2(2 l+3)}} \exp \left(i\left(\omega_{l}+\omega_{l+2}\right)\right) U_{3 / 2(l+2) 1 / 2 l}^{l+1 / 2} P_{l+2}^{0} \\
& -\frac{l \sqrt{3(l-1)}}{\sqrt{2(2 l-1)}} \exp \left(i\left(\omega_{l}+\omega_{l-2}\right)\right) U_{3 / 2(l-2) 1 / 2 l}^{l-1 / 2} P_{l-2}^{0} \\
& +\frac{l \sqrt{l-1}}{\sqrt{2(2 l+1)}} \exp \left(i\left(\omega_{l}+\omega_{l-1}\right)\right) U_{3 / 2(l-1) 1 / 2 l}^{l-1 / 2} P_{l-1}^{0} \\
& +\frac{l \sqrt{l+1}}{\sqrt{2(2 l-1)}} \exp \left(2 i \omega_{l}\right) U_{3 / 2 l 1 / 2 l}^{l-1 / 2} P_{l}^{0} \\
& \left.-\frac{l \sqrt{3(l+1)}}{\sqrt{2(2 l+1)}} \exp \left(i\left(\omega_{l}+\omega_{l+1}\right)\right) U_{3 / 2(l+1) 1 / 2 l}^{l-1 / 2} P_{l+1}^{0}\right] \\
& \mathfrak{M}_{3 / 2 \quad 1 / 2}^{1 / 2-1 / 2}=-\frac{i}{2 k} \sum_{l}\left[\frac{\sqrt{3 l(l-1)}(l+1)}{\sqrt{2(2 l+1)}} \exp \left(i\left(\omega_{l}+\omega_{l-1}\right)\right) U_{3 / 2(l-1) 1 / 2 l}^{l+1 / 2} P_{l-1}^{0}\right. \\
& +\frac{(l+1) \sqrt{l+3}}{\sqrt{2(2 l+3)}} \exp \left(2 i \omega_{l}\right) U_{3 / 2 l 1 / 2 l}^{l+1 / 2} P_{l}^{0} \\
& -\frac{(l-1) \sqrt{l+1}}{\sqrt{2(2 l+3)}} \exp \left(i\left(\omega_{l}+\omega_{l+1}\right)\right) U_{3 / 2(l+1) 1 / 2 l}^{l+1 / 2} P_{l+1}^{0} \\
& -\frac{(l+1) \sqrt{3(l+3)}}{\sqrt{2(2 l+3)}} \exp \left(i\left(\omega_{l}+\omega_{l+2}\right)\right) U_{3 / 2(l+2) 1 / 2 l}^{l+1 / 2} P_{l+2}^{0} \\
& -\frac{l \sqrt{3(l-2)}}{\sqrt{2(2 l-1)}} \exp \left(i\left(\omega_{l}+\omega_{l-2}\right)\right) U_{3 / 2(l-2) 1 / 2 l}^{l-1 / 2} P_{l-2}^{0} \\
& -\frac{(l+1) \sqrt{l}}{\sqrt{2(2 l+1)}} \exp \left(i\left(\omega_{l}+\omega_{l-1}\right)\right) U_{3 / 2(l-1) 1 / 2 l}^{l-1 / 2} P_{l-1}^{0} \\
& +\frac{(l-2) \sqrt{l}}{\sqrt{2(2 l-1)}} \exp \left(2 i \omega_{l}\right) U_{3 / 2(l-1) 1 / 2 l}^{l-1 / 2} P_{l-1}^{0} \\
& \left.+\frac{l \sqrt{3(l+2)}}{\sqrt{2(2 l+1)}} \exp \left(i\left(\omega_{l}+\omega_{l+1}\right)\right) U_{3 / 2(l+1) 1 / 2 l}^{l-1 / 2} P_{l+1}^{0}\right]
\end{aligned}
$$




$$
\begin{aligned}
& \mathfrak{M}_{1 / 2}^{3 / 2} 3 / 2=-\frac{i}{2 k} \sum_{l}\left[\frac{l+3}{\sqrt{2(2 l+3)(l+1)}} \exp \left(i\left(\omega_{l}+\omega_{l+1}\right)\right) U_{1 / 2(l+1) 3 / 2 l}^{l+3 / 2} P_{l+1}^{1}\right. \\
& -\sqrt{\frac{l+1}{2(2 l+3)}} \exp \left(i\left(\omega_{l}+\omega_{l+2}\right)\right) U_{1 / 2(l+2) 3 / 2 l}^{l+3 / 2} P_{l+2}^{1} \\
& -\frac{l+2}{\sqrt{2 l(2 l+3)}} \exp \left(2 i \omega_{l}\right) U_{1 / 2 l 3 / 2 l}^{l+1 / 2} P_{l}^{1} \\
& +\sqrt{\frac{3 l}{2(2 l+3)}} \exp \left(i\left(\omega_{l}+\omega_{l+1}\right)\right) U_{1 / 2(l+1) 3 / 2 l}^{l+1 / 2} P_{l+1}^{1} \\
& +\sqrt{\frac{l+1}{2(2 l-1)}} \exp \left(i\left(\omega_{l}+\omega_{l-1}\right)\right) U_{1 / 2(l-1) 3 / 2 l}^{l-1 / 2} P_{l-1}^{1} \\
& -\frac{l-1}{\sqrt{2(l+1)(2 l-1)}} \exp \left(2 i \omega_{l}\right) U_{1 / 2 l 3 / 2 l}^{l-1 / 2} P_{l}^{1} \\
& -\sqrt{\frac{l}{2(2 l-1)}} \exp \left(i\left(\omega_{l}+\omega_{l-2}\right)\right) U_{1 / 2(l-2) 3 / 2 l}^{l-3 / 2} P_{l-2}^{1} \\
& \left.+\frac{l-2}{\sqrt{2 l(2 l-1)}} \exp \left(i\left(\omega_{l}+\omega_{l-1}\right)\right) U_{1 / 2(l-1) 3 / 2 l}^{l-3 / 2} P_{l}^{1}\right] \\
& \mathfrak{M}_{1 / 21 / 2}^{3 / 21 / 2}=-\frac{i}{2 k} \sum_{l}\left[\frac{(l+2) \sqrt{l+3}}{\sqrt{2(2 l+3)}} \exp \left(i\left(\omega_{l}+\omega_{l+1}\right)\right) U_{1 / 2(l+1) 3 / 2 l}^{l+3 / 2} P_{l+1}^{0}\right. \\
& -\frac{(l+2) \sqrt{l+3}}{\sqrt{2(2 l+3)}} \exp \left(i\left(\omega_{l}+\omega_{l+2}\right)\right) U_{1 / 2(l+2) 3 / 2 l}^{l+3 / 2} P_{l+2}^{0} \\
& -\frac{(l+1) \sqrt{3(l+2)}}{\sqrt{2(2 l+3)}} \exp \left(2 i \omega_{l}\right) U_{1 / 2(l) 3 / 2 l}^{l+1 / 2} P_{l}^{0} \\
& +\frac{(l+1) \sqrt{3(l+2)}}{\sqrt{2(2 l+3)}} \exp \left(i\left(\omega_{l}+\omega_{l+1}\right)\right) U_{1 / 2(l+1) 3 / 2 l}^{l+1 / 2} P_{l+1}^{0} \\
& +\frac{l \sqrt{3(l-1)}}{\sqrt{2(2 l-1)}} \exp \left(i\left(\omega_{l}+\omega_{l-1}\right)\right) U_{1 / 2(l-1) 3 / 2 l}^{l-1 / 2} P_{l-1}^{0} \\
& +\frac{l \sqrt{3(l-1)}}{\sqrt{2(2 l-1)}} \exp \left(2 i \omega_{l}\right) U_{1 / 2 l) 3 / 2 l}^{l-1 / 2} P_{l}^{0} \\
& -\frac{(l-1) \sqrt{(l-2)}}{\sqrt{2(2 l-1)}} \exp \left(i\left(\omega_{l}+\omega_{l-2}\right)\right) U_{1 / 2(l-2) 3 / 2 l}^{l-3 / 2} P_{l-2}^{0} \\
& \left.+\frac{(l-1) \sqrt{(l-2)}}{\sqrt{2(2 l-1)}} \exp \left(i\left(\omega_{l}+\omega_{l-1}\right)\right) U_{1 / 2(l-1) 3 / 2 l}^{l-3 / 2} P_{l-1}^{0}\right]
\end{aligned}
$$




$$
\begin{aligned}
& \mathfrak{M}_{1 / 2}^{3 / 2-1 / 2}=-\frac{i}{2 k} \sum_{l}\left[\sqrt{\frac{l+3}{2(2 l+3)}} \exp \left(i\left(\omega_{l}+\omega_{l+1}\right)\right) U_{1 / 2(l+1) 3 / 2 l}^{l+3 / 2} P_{l+1}^{1}\right. \\
& +\sqrt{\frac{l+3}{2(2 l+3)}} \exp \left(i\left(\omega_{l}+\omega_{l+2}\right)\right) U_{1 / 2(l+2) 3 / 2 l}^{l+3 / 2} P_{l+2}^{1} \\
& -\sqrt{\frac{3(l+2)}{2(2 l+3)}} \exp \left(2 i \omega_{l}\right) U_{1 / 2(l) 3 / 2 l}^{l+1 / 2} P_{l}^{1} \\
& -\sqrt{\frac{3(l+2)}{2(2 l+3)}} \exp \left(i\left(\omega_{l}+\omega_{l+1}\right)\right) U_{1 / 2(l+1) 3 / 2 l}^{l+1 / 2} P_{l+1}^{1} \\
& +\frac{\sqrt{3(l-1)}}{\sqrt{2(2 l-1)}} \exp \left(i\left(\omega_{l}+\omega_{l-1}\right)\right) U_{1 / 2(l-1) 3 / 2 l}^{l-1 / 2} P_{l-1}^{1} \\
& +\sqrt{\frac{3(l-1)}{2(2 l-1)}} \exp \left(2 i \omega_{l}\right) U_{1 / 2 l) 3 / 2 l}^{l-1 / 2} P_{l}^{1} \\
& -\sqrt{\frac{(l-2)}{2(2 l-1)}} \exp \left(i\left(\omega_{l}+\omega_{l-2}\right)\right) U_{1 / 2(l-2) 3 / 2 l}^{l-3 / 2} P_{l-2}^{1} \\
& \left.+\sqrt{\frac{(l-2)}{2(2 l-1)}} \exp \left(i\left(\omega_{l}+\omega_{l-1}\right)\right) U_{1 / 2(l-1) 3 / 2 l}^{l-3 / 2} P_{l-1}^{1}\right] \\
& \mathfrak{M}_{1 / 21 / 2}^{3 / 2-3 / 2}=\frac{i}{2 k} \sum_{l}\left[\frac{1}{\sqrt{2 l(2 l+3)(l+1)}} \exp \left(i\left(\omega_{l}+\omega_{l+1}\right)\right) U_{1 / 2(l+1) 3 / 2 l}^{l+3 / 2} P_{l+1}^{2}\right. \\
& +\frac{1}{\sqrt{2 l(2 l+3)(l+1)}} \exp \left(i\left(\omega_{l}+\omega_{l+2}\right)\right) U_{1 / 2(l+2) 3 / 2 l}^{l+3 / 2} P_{l+2}^{2} \\
& -\sqrt{\frac{3}{2 l(2 l+3)}} \exp \left(2 i \omega_{l}\right) U_{1 / 2(l) 3 / 2 l}^{l+1 / 2} P_{l}^{2} \\
& -\sqrt{\frac{3}{2 l(2 l+3)}} \exp \left(i\left(\omega_{l}+\omega_{l+1}\right)\right) U_{1 / 2(l+1) 3 / 2 l}^{l+1 / 2} P_{l+1}^{2} \\
& +\sqrt{\frac{3}{2(2 l-1)(l+1)}} \exp \left(i\left(\omega_{l}+\omega_{l-1}\right)\right) U_{1 / 2(l-1) 3 / 2 l}^{l-1 / 2} P_{l-1}^{2} \\
& +\sqrt{\frac{3}{2(2 l-1)(l+1)}} \exp \left(2 i \omega_{l}\right) U_{1 / 2 l) 3 / 2 l}^{l-1 / 2} P_{l}^{2} \\
& -\frac{1}{\sqrt{2 l(2 l-1)}} \exp \left(i\left(\omega_{l}+\omega_{l-2}\right)\right) U_{1 / 2(l-2) 3 / 2 l}^{l-3 / 2} P_{l-2}^{2} \\
& \left.-\frac{1}{\sqrt{2 l(2 l-1)}} \exp \left(i\left(\omega_{l}+\omega_{l-1}\right)\right) U_{1 / 2(l-1) 3 / 2 l}^{l-3 / 2} P_{l-1}^{2}\right]
\end{aligned}
$$

Appendix C: Partial amplitudes for the channel spin $S=5 / 2$ 


$$
\begin{aligned}
\mathcal{S}^{5 / 2} 1 / 2= & f_{c}(\theta)+\frac{1}{4 i k} \sum_{l=0}\left[\frac{5(l+1)(l+2)(l+3)}{(2 l+3)(2 l+5)} \alpha^{l+5 / 2}+\frac{l(l+1)(l+2)}{(2 l+3)(2 l+5)} \alpha^{l+3 / 2}+\frac{2 l(l+1)(l+2)}{(2 l+3)(2 l-1)} \alpha^{l+1 / 2}\right. \\
& \left.+\frac{2 l(l+1)(l-1)}{(2 l+3)(2 l-1)} \alpha^{l-1 / 2}+\frac{l(l+1)(l-1)}{(2 l-1)(2 l-3)} \alpha^{l-3 / 2}+\frac{5 l(l-1)(l-2)}{(2 l-3)(2 l-1)} \alpha^{l-5 / 2}\right] \\
& \times \exp \left(2 i \omega_{l}\right) P_{l}(\cos \theta) \\
\mathcal{S}^{5 / 23 / 2}= & f_{c}(\theta)+\frac{1}{8 i k} \sum_{l=0}^{3 / 2}\left[\frac{5(l+1)(l+3)(l+4)}{(2 l+3)(2 l+5)} \alpha^{l+5 / 2}+\frac{9 l(l+2)(l+3)}{(2 l+3)(2 l+5)} \alpha^{l+3 / 2}\right. \\
& +\frac{2 l(l+1)(l-3)^{2}}{(2 l+3)(2 l-1)} \alpha^{l+1 / 2}+\frac{2 l(l+4)^{2}}{(2 l+3)(2 l-1)} \alpha^{l-1 / 2}+\frac{9(l+1)(l-1)(l-2)}{(2 l-1)(2 l-3)} \alpha^{l-3 / 2} \\
& \left.+\frac{5 l(l-2)(l-3)}{(2 l-3)(2 l-1)} \alpha^{l-5 / 2}\right] \operatorname{exp~}\left(2 i \omega_{l}\right) P_{l}(\cos \theta)
\end{aligned}
$$

$$
\begin{aligned}
\mathcal{S}_{5 / 2}^{5 / 2}= & f_{c}(\theta)+\frac{1}{8 i k} \sum_{l=0}\left[\frac{(l+3)(l+4)(l+5)}{(2 l+3)(2 l+5)} \alpha^{l+5 / 2}+\frac{5(l+2)(l+3)(l+4)}{(2 l+3)(2 l+5)} \alpha^{l+3 / 2}\right. \\
& +\frac{10(l+1)(l-1)(l+3)}{(2 l+3)(2 l-1)} \alpha^{l+1 / 2}+\frac{10 l(l+2)(l-2)}{(2 l+3)(2 l-1)} \alpha^{l-1 / 2}+\frac{5(l-1)(l-2)(l-3)}{(2 l-1)(2 l-3)} \alpha^{l-3 / 2}
\end{aligned}
$$$$
\left.+\frac{(l-2)(l-3)(l-4)}{(2 l-3)(2 l-1)} \alpha^{l-5 / 2}\right] \exp \left(2 i \omega_{l}\right) P_{l}(\cos \theta) \text {, }
$$

$$
\begin{aligned}
\mathcal{S}_{-1 / 2}^{5 / 2}= & -\frac{1}{4 i k} \sum_{l=1}\left[\frac{5(l+2)(l+3)}{(2 l+3)(2 l+5)} \alpha^{l+5 / 2}-\frac{(l+2)(l+5)}{(l+3)(2 l+5)} \alpha^{l+3 / 2}+\frac{2(l+2)(l-2)}{(2 l+3)(2 l-1)} \alpha^{l+1 / 2}\right. \\
& \left.-\frac{2(l+3)(l-1)}{(2 l+3)(2 l-1)} \alpha^{l-1 / 2}+\frac{(l-1)(l-4)}{(2 l-1)(2 l-3)} \alpha^{l-3 / 2}-\frac{5(l-1)(l-2)}{(2 l-3)(2 l-1)} \alpha^{l-5 / 2}\right] \\
& \times \exp \left(2 i \omega_{l}\right) P_{l}^{1}(\cos \theta),
\end{aligned}
$$

$$
\begin{aligned}
\mathcal{S}_{3 / 2}^{5 / 2}= & -\frac{\sqrt{2}}{8 i k} \sum_{l=1}\left[\frac{5(l+2)(l+3)}{(2 l+3)(2 l+5)} \alpha^{l+5 / 2}+\frac{(l+2)(3 l+5)}{(2 l+3)(2 l+5)} \alpha^{l+3 / 2}-\frac{2(l+2)(l+1)}{(2 l+3)(2 l-1)} \alpha^{l+1 / 2}\right. \\
& \left.+\frac{2 l(l-1)}{(2 l+3)(2 l-1)} \alpha^{l-1 / 2}-\frac{(l-1)(3 l-2)}{(2 l-1)(2 l-3)} \alpha^{l-3 / 2}-\frac{5(l-1)(l-2)}{(2 l-3)(2 l-1)} \alpha^{l-5 / 2}\right] \\
& \times \exp \left(2 i \omega_{l}\right) P_{l}^{1}(\cos \theta)
\end{aligned}
$$

$$
\begin{aligned}
\mathcal{S}^{5 / 2}{ }_{1 / 2}^{3 / 2}= & -\frac{\sqrt{2}}{8 i k} \sum_{l=1}\left[\frac{5(l+3)(l+4)}{(2 l+3)(2 l+5)} \alpha^{l+5 / 2}+\frac{3(l+2)(l+3)(l-5)}{(l+1)(2 l+3)(2 l+5)} \alpha^{l+3 / 2}-\frac{2\left(l^{2}+4 l-6\right)(l-3)}{l(2 l+3)(2 l-1)} \alpha^{l+1 / 2}\right. \\
& \left.+\frac{2 l(l+4)\left(l^{2}-2 l-9\right)}{(l+1)(2 l+3)(2 l-1)} \alpha^{l-1 / 2}-\frac{3(l+6)(l-1)(l-2)}{l(2 l-1)(2 l-3)} \alpha^{l-3 / 2}-\frac{5(l-2)(l-3)}{(2 l-3)(2 l-1)} \alpha^{l-5 / 2}\right] \\
& \times \exp \left(2 i \omega_{l}\right) P_{l}^{1}(\cos \theta),
\end{aligned}
$$

$$
\begin{aligned}
\mathcal{S}_{5 / 2}^{5 / 2}= & -\frac{\sqrt{5}}{8 i k} \sum_{l=1}\left[\frac{(l+3)(l+4)}{(2 l+3)(2 l+5)} \alpha^{l+5 / 2}+\frac{3(l+2)(l+3)}{(2 l+3)(2 l+5)} \alpha^{l+3 / 2}+\frac{2(l+1)(l-3)}{(2 l+3)(2 l-1)} \alpha^{l+1 / 2}\right. \\
& \left.-\frac{2 l(l+4)}{(2 l+3)(2 l-1)} \alpha^{l-1 / 2}-\frac{3(l-1)(l-2)}{(2 l-1)(2 l-3)} \alpha^{l-3 / 2}-\frac{(l-2)(l-3)}{(2 l-3)(2 l-1)} \alpha^{l-5 / 2}\right] \\
& \times \exp \left(2 i \omega_{l}\right) P_{l}^{1}(\cos \theta)
\end{aligned}
$$




$$
\begin{aligned}
& \mathcal{S}_{3 / 2}^{5 / 2}=-\frac{\sqrt{5}}{8 i k} \sum_{l=1}\left[\frac{(l+3)(l+4)(l+5)}{(l+1)(2 l+3)(2 l+5)} \alpha^{l+5 / 2}+\frac{(l+2)(l+3)(l+4)(3 l-5)}{l(l+1)(2 l+3)(2 l+5)} \alpha^{l+3 / 2}\right. \\
& +\frac{2(l+3)(l-1)(l-8)}{l(2 l+3)(2 l-1)} \alpha^{l+1 / 2}-\frac{2(l+9)(l+2)(l-2)}{(l+1)(2 l+3)(2 l-1)} \alpha^{l-1 / 2}-\frac{(3 l+8)(l-1)(l-2)(l-3)}{l(l+1)(2 l-1)(2 l-3)} \alpha^{l-3 / 2} \\
& \left.-\frac{(l-2)(l-3)(l-4)}{l(2 l-3)(2 l-1)} \alpha^{l-5 / 2}\right] \exp \left(2 i \omega_{l}\right) P_{l}^{1}(\cos \theta) \\
& \mathcal{S}_{3 / 2}^{5 / 2-1 / 2}=\frac{\sqrt{2}}{8 i k} \sum_{l=2}\left[\frac{5(l+3)}{(2 l+3)(2 l+5)} \alpha^{l+5 / 2}-\frac{3 l+10}{(2 l+3)(2 l+5)} \alpha^{l+3 / 2}-\frac{2(l+7)}{(2 l+3)(2 l-1)} \alpha^{l+1 / 2}\right. \\
& \left.-\frac{2(l-6)}{(2 l+3)(2 l-1)} \alpha^{l-1 / 2}-\frac{3 l-7}{(2 l-1)(2 l-3)} \alpha^{l-3 / 2}+\frac{5(l-2)}{(2 l-3)(2 l-1)} \alpha^{l-5 / 2}\right] \\
& \times \exp \left(2 i \omega_{l}\right) P_{l}^{2}(\cos \theta) \text {, } \\
& \mathcal{S}_{5 / 2}^{5 / 2} \underset{5 / 2}{1 / 2}=\frac{\sqrt{10}}{8 i k} \sum_{l=2}\left[\frac{l+3}{(2 l+3)(2 l+5)} \alpha^{l+5 / 2}+\frac{l+2}{(2 l+3)(2 l+5)} \alpha^{l+3 / 2}-\frac{2(l+1)}{(2 l+3)(2 l-1)} \alpha^{l+1 / 2}\right. \\
& \left.-\frac{2 l}{(2 l+3)(2 l-1)} \alpha^{l-1 / 2}+\frac{l-1}{(2 l-1)(2 l-3)} \alpha^{l-3 / 2}+\frac{l-2}{(2 l-3)(2 l-1)} \alpha^{l-5 / 2}\right] \\
& \times \exp \left(2 i \omega_{l}\right) P_{l}^{2}(\cos \theta) \text {, } \\
& \mathcal{S}_{1 / 2}^{5 / 2-3 / 2}=\frac{1}{4 \sqrt{2} i k} \sum_{l=2}\left[\frac{5(l+3)(l+4)}{(l+2)(2 l+3)(2 l+5)} \alpha^{l+5 / 2}-\frac{3(l+3)(l+10)}{(l+1)(2 l+3)(2 l+5)} \alpha^{l+3 / 2}\right. \\
& -\frac{2(l-3)\left(l^{2}-2 l-18\right)}{l(l+2)(2 l+3)(2 l-1)} \alpha^{l+1 / 2}-\frac{2(l+4)\left(l^{2}+4 l-15\right)}{(l+1)(l-1)(2 l+3)(2 l-1)} \alpha^{l-1 / 2}-\frac{3(l-2)(l-9)}{l(2 l-1)(2 l-3)} \alpha^{l-3 / 2} \\
& \left.+\frac{5(l-2)(l-3)}{(l-1)(2 l-3)(2 l-1)} \alpha^{l-5 / 2}\right] \exp \left(2 i \omega_{l}\right) P_{l}^{2}(\cos \theta)
\end{aligned}
$$$$
\mathcal{S}_{1 / 2}^{5 / 2}=\frac{\sqrt{10}}{8 i k} \sum_{l=2}\left[\frac{(l+3)(l+4)(l+5)}{(l+1)(l+2)(2 l+3)(2 l+5)} \alpha^{l+5 / 2}+\frac{(l+3)(l+4)(l-10)}{l(l+1)(2 l+3)(2 l+5)} \alpha^{l+3 / 2}\right.
$$$$
-\frac{2(l+3)\left(l^{2}+6 l-22\right)}{l(l+2)(2 l+3)(2 l-1)} \alpha^{l+1 / 2}-\frac{2(l-2)\left(l^{2}-4 l-27\right)}{(l+1)(l-1)(2 l+3)(2 l-1)} \alpha^{l-1 / 2}+\frac{(l-2)(l-3)(l+11)}{l(l+1)(2 l-1)(2 l-3)} \alpha^{l-3 / 2}
$$$$
\left.+\frac{(l-2)(l-3)(l-4)}{l(l-1)(2 l-3)(2 l-1)} \alpha^{l-5 / 2}\right] \exp \left(2 i \omega_{l}\right) P_{l}^{2}(\cos \theta)
$$$$
\mathcal{S}_{-5 / 2}^{5 / 2}=-\frac{\sqrt{10}}{8 i k} \sum_{l=3}\left[\frac{1}{(2 l+3)(2 l+5)} \alpha^{l+5 / 2}-\frac{1}{(2 l+3)(2 l+5)} \alpha^{l+3 / 2}-\frac{2}{(2 l+3)(2 l-1)} \alpha^{l+1 / 2}\right.
$$$$
\left.+\frac{2}{(2 l+3)(2 l-1)} \alpha^{l-1 / 2}+\frac{1}{(2 l-1)(2 l-3)} \alpha^{l-3 / 2}-\frac{1}{(2 l-3)(2 l-1)} \alpha^{l-5 / 2}\right]
$$$$
\times \exp \left(2 i \omega_{l}\right) P_{l}^{3}(\cos \theta) \text {, }
$$

$$
\begin{aligned}
\mathcal{S}_{-3 / 2}^{5 / 23 / 2}= & -\frac{1}{8 i k} \sum_{l=3}\left[\frac{5(l+4)}{(l+2)(2 l+3)(2 l+5)} \alpha^{l+5 / 2}-\frac{9(l+5)}{(l+1)(2 l+3)(2 l+5)} \alpha^{l+3 / 2}\right. \\
& +\frac{2(l+12)(l-3)}{l(l+2)(2 l+3)(2 l-1)} \alpha^{l+1 / 2}-\frac{2(l+4)(l-11)}{(l+1)(l-1)(2 l+3)(2 l-1)} \alpha^{l-1 / 2}+\frac{9(l-4)}{l(2 l-1)(2 l-3)} \alpha^{l-3 / 2} \\
& \left.-\frac{5(l-3)}{(l-1)(2 l-3)(2 l-1)} \alpha^{l-5 / 2}\right] \exp \left(2 i \omega_{l}\right) P_{l}^{3}(\cos \theta)
\end{aligned}
$$




$$
\begin{aligned}
& \mathcal{S}_{-1 / 2}^{5 / 25 / 2}=-\frac{\sqrt{10}}{8 i k} \sum_{l=3}\left[\frac{(l+4)(l+5)}{(l+1)(l+2)(2 l+3)(2 l+5)} \alpha^{l+5 / 2}-\frac{(l+4)(l+15)}{l(l+1)(2 l+3)(2 l+5)} \alpha^{l+3 / 2}\right. \\
&-\frac{2\left(l^{2}-4 l-42\right)}{l(l+2)(2 l+3)(2 l-1)} \alpha^{l+1 / 2}+\frac{2\left(l^{2}+6 l-37\right)}{(l+1)(l-1)(2 l+3)(2 l-1)} \alpha^{l-1 / 2}+\frac{(l-3)(l-14)}{l(l+1)(2 l-1)(2 l-3)} \alpha^{l-3 / 2} \\
&\left.-\frac{(l-3)(l-4)}{l(l-1)(2 l-3)(2 l-1)} \alpha^{l-5 / 2}\right] \exp \left(2 i \omega_{l}\right) P_{l}^{3}(\cos \theta), \\
& \mathcal{S}^{5 / 2-3 / 2}= \frac{5}{8 i k} \sum_{l=4}\left[\frac{1}{(l+2)(2 l+3)(2 l+5)} \alpha^{l+5 / 2}-\frac{3}{(l+1)(2 l+3)(2 l+5)} \alpha^{l+3 / 2}+\frac{2(l-3)}{l(l+2)(2 l+3)(2 l-1)} \alpha^{l+1 / 2}\right. \\
&\left.+\frac{2(l+4)}{(l+1)(l-1)(2 l+3)(2 l-1)} \alpha^{l-1 / 2}-\frac{3}{l(2 l-1)(2 l-3)} \alpha^{l-3 / 2}+\frac{1}{(l-1)(2 l-3)(2 l-1)} \alpha^{l-5 / 2}\right] \\
& \times \exp \left(2 i \omega_{l}\right) P_{l}^{4}(\cos \theta), \\
& \mathcal{S}^{5 / 2-5 / 2}= \frac{5}{8 i k} \sum_{l=4}\left[\frac{2 l 6)}{(l+1)(l+2)(2 l+3)(2 l+5)} \alpha^{l+5 / 2}-\frac{3 l+20}{l(l+1)(2 l+3)(2 l+5)} \alpha^{l+3 / 2}\right. \\
&+\frac{2(l+17)}{l(l+2)(2 l+3)(2 l-1)} \alpha^{l+1 / 2}+\frac{2(l-16)}{(l+1)(l-1)(2 l+3)(2 l-1)} \alpha^{l-1 / 2}-\frac{3 l-17}{l(l+1)(2 l-1)(2 l-3)} \alpha^{l-3 / 2} \\
&\left.+\frac{l-4}{l(l-1)(2 l-3)(2 l-1)} \alpha^{l-5 / 2}\right] \exp \left(2 i \omega_{l}\right) P_{l}^{4}(\cos \theta), \\
& \mathcal{S}_{-5 / 2}^{5 / 25 / 2}=\left.-\frac{1}{2 i k} \sum_{l=5} \sqrt{\frac{(l-5) !}{(l+5) !}} \alpha^{l+5 / 2}-5 \alpha^{l+3 / 2}+10 \alpha^{l+1 / 2}-10 \alpha^{l-1 / 2}+5 \alpha^{l-3 / 2}-\alpha^{l-5 / 2}\right] \exp \left(2 i \omega_{l}\right) P_{l}^{5}(\cos \theta) . \\
&(\mathrm{C} 18)
\end{aligned}
$$

[1] S. B. Dubovichenko, Radiative Neutron Capture. Walter de Gruyter GmbH, Berlin/Boston, 296 p. (2019).

[2] S. Quaglioni, P. Navrátil, and R. Roth, EPJ Web of Conf. 3, 01012 (2010).

[3] H. Friedrich, Scattering theory, Springer, Heidelberg, 287 p. (2013).

[4] E. Henley, Subatomic physics, 3d edition, World Scientific Publishing Co. Pte. Ltd., Singapore, 2007,620 p.

[5] P. E. Hodgson, The optical model of elastic scattering. Clarendon Press, Oxford, 211 p. (1963).

[6] S. B. Dubovichenko, N. A.Burkova, A. V. Dzhazairov-Kakhramanova, R. Ya. Kezerashvili, Ch. T. Omarov, A. S. Tkachenko, and D. M. Zazulin, Nucl. Phys. A 987, 46 (2019).

[7] C. A. Barnes, D. D. Clayton, and D. N. Schramm, Essays in Nuclear Astrophysics. Presented to William A. Fowler. Cambridge University Press, UK, 562 p. (1982).

[8] F. Iocco, G. Manganob, G. Miele, O. Pisanti, and P. D. Serpico, Physics Reports 472, 1 (2009).

[9] K. Fang, J. Zou, H. He, Q. Wang, J. Zhao, T. Wang, and J. Kasagi, Phys. Rew. C 94, 054602 (2016).

[10] J. J. He, S. Z. Chena, C. E. Rolfs et al., Phys. Let. B 725, 287 (2013).

[11] A. Coc, S. Goriely, Yi Xu, M. Saimpert, and E. Vangioni, The Astrophysical Journal 744, 158 (2012).

[12] LUNA Collaboration: M. Anders, D. Trezzi, R. Menegazzo et al., Phys. Rev. Lett. 113, 042501 (2014).

[13] E. Hiyama and M. Kamimura, Front. Phys. 13, 132106 (2018).

[14] A. Gnech and L. E. Marcucci, Nucl. Phys. A 987, 1 (2019).

[15] F. Nichitiu, Phys. Part. Nucl. 12, 805 (1981).

[16] F. Nichitiu, Phase shifts analysis in physics, Acad. Publ., Romania, 416 p. (1980).

[17] S. B. Dubovichenko, Phase shifts analysis in nuclear astrophysics, Lambert Academy Publ. GmbH\&Co, Germany, 368 p. (2015).

[18] G. R. Satchler, Direct nuclear reactions, Oxford University Press, UK, 833 p. (2009).

[19] R. G. Seyler, Nucl. Phys. A 124, 253 (1969).

[20] L. Brown, E. Stainer, L. G. Arnold, and R.G. Seyler, Nucl. Phys. A 206, 353 (1973).

[21] A. S. Tkachenko, N. A. Burkova, and S. B. Dubovichenko, Isv. Vuzov, Fizika 62, 46 (2019). 
[22] A. M. Lane and R. G. Thomas, Rev. Mod. Phys. 30, 257 (1958).

[23] D. A. Varshalovich, A. N. Moskalev, and V. K. Khersonskii, Quantum theory of angular momentum and its applications, V1, FIZMATLIT, Moscow, 568 p. (2017).

[24] S. B. Dubovichenko, Methods for calculating nuclear characteristics. Models - methods - programs, KazATiSO, Almaty, 311 p. (2006). 